\title{
Embryonic diapause in the short-tailed fruit bat, Carollia perspicillata: why this is postimplantational
}

\author{
JJ Rasweiler IV ${ }^{1,2}$ and NK Badwaik ${ }^{\mathbf{1}}$ \\ ${ }^{1}$ Department of Obstetrics \& Gynecology, Weill Medical College of Cornell University, New \\ York, USA \\ ${ }^{2}$ Department of Obstetrics \& Gynecology, State University of New York Downstate \\ Medical Center, New York, USA
}

Corresponding author e-mail:john.rasweiler.iv@gmail.com

\begin{abstract}
Pregnancy has been studied in Carollia perspicillata bred in captivity or collected from a reproductively-synchronized wild population on Trinidad, West Indies. In both situations, periods of postimplantational embryonic diapause were sometimes observed. In captivity, this was induced by stress, or once-weekly periods of food deprivation, and resulted in gestation periods of highly variable duration (105-237 days). The normal gestation period for this species is 113-120 days. In the wild, Carollia exhibits two synchronized pregnancy periods. One includes a diapause of at least 4450 days, but probably somewhat longer, while the other is of normal length. The diapause occurs at the primitive streak stage of development. The postimplantational timing of the diapause is associated with multiple other reproductive specializations, all of which seem to be intended to facilitate blastocyst attachment very soon after entry into the uterus, within a preferred implantation zone. Blastocysts of this species were never observed to have passed that zone or to have a prolonged, free-floating period in the main uterine cavity. This facilitates placental development within an optimally vascularized part of the uterus and the eventual production of a large, highly precocious infant. Comparative immunocytochemical and ultrastructural studies of normal versus delayed pregnancies suggest that inadequate trophoblastic differentiation within the developing placenta may play an important role in maintaining the diapause. In part this may be responsible for delayed invasion of the embryonic side of the placenta by vascularized allantoic mesenchyme. The diapause is also associated with greatly increased trophoblastic invasiveness of unknown significance.
\end{abstract}

\section{Introduction}

Embryonic diapause has now been observed in more than 130 species of mammals belonging to 10 different orders [1-3]. In most cases, this occurs prior to implantation, at the blastocyst stage of development. In some species of bats, however, the diapause occurs after implantation [4-6]. In an effort to distinguish the two forms, the postimplantational version has generally been referred to as "delayed development". Choice of the latter terminology seems unfortunate, as both forms clearly involve developmental delays; however, its use is now well-entrenched in the literature.

Most cases of a postimplantational diapause have been documented for a handful of species belonging to the large chiropteran families Phyllostomidae (about 192 described species) and 
Pteropodidae (about 187 species) [5]. Because these families are tropical or subtropical in distribution, the reproductive seasonality of most members has never been thoroughly studied. This raises the possibility that more may eventually be found to exhibit a diapause. Further studies of their reproductive biology seem warranted, because these are highly successful mammalian families in terms of species diversity and numerical abundance. Many members also play important ecological roles by pollinating and/or disseminating the seeds of native plants $[7,8]$.

In no case has the environmental cue (zeitgeber) responsible for synchronizing the occurrence of the diapause in these seasonally-breeding, tropical mammals been identified. Changes in daylength provide reliable cues controlling the breeding seasonality of many temperate latitude mammals, but these are less pronounced or absent close to the Equator [9]. That does not mean that changing daylength can be completely discounted, especially as other potential environmental cues (e.g., the timing of rainy and dry seasons) often vary substantially from year to year.

The occurrence of a postimplantational embryonic diapause has now been well-documented for the short-tailed fruit bat (Carollia perspicillata; family Phyllostomidae), which is probably the most abundant mammal inhabiting forested areas in the lowland tropics of the New World $[4,10]$. This was first suggested by the timing of birth periods in a reproductively-synchronized wild population living in French Guiana [11]. It was subsequently confirmed by carefully-timed histological and embryonic staging studies of both wild and captive-bred Carollia [4]. These findings are reviewed below.

Carollia initially became of interest, because it exhibits multiple female reproductive similarities to humans. These include being monovular, having a simplex uterus, exhibiting true menstruation, displaying interstitial implantation of the blastocyst in a preferred portion of the uterus, and having highly invasive trophoblast $[10,12,13]$. During the course of efforts to more fully evaluate the species' potential utility as a model for the study of human reproduction, it was noted that some other aspects of its reproductive biology are quite unusual. It now seems that these latter specializations, as well as the postimplantational diapause, all probably evolved to ensure the birth of large, highly precocious young. Such young should presumably have a higher probability of surviving the dangers of early postnatal life. The adaptive significance of the unique, postimplantational timing of this diapause in some bats has long been a puzzle. This can now hopefully be resolved.

There is also a notable difference between the pre- and postimplantational forms of diapause that has attracted too little research attention. In the case of the latter, physiological exchange between mother and embryo is always mediated by a placenta. This suggests that altered placental development and function may play important roles in triggering and controlling the postimplantational diapause. For that reason, species exhibiting this phenomenon may provide novel models for better understanding early placentation. This will be more fully explored below.

\section{Discovery of embryonic diapause in captive Carollia}

The discovery of a diapause during pregnancy in Carollia occurred serendipitously, during the course of studies that intended to focus upon blastocyst implantation and trophoblast-maternal vascular interactions. In order to pursue these studies, two large groups of bats were captured in the wild on the West Indian island of Trinidad in November 1991 and October 1993 and brought to New York to stock an experimental breeding colony. In both cases, the sexes were initially housed in sexually-segregated groups for a number of months, to provide time for adjustment to captivity and for any females pregnant from the wild to complete their pregnancies. 
As some Carollia exhibited interbirth intervals of as little as $105-125$ days in previous studies [14, 15], it was not anticipated that any of the females would give birth more than about 125 days after being sexually-isolated. Surprisingly, many in the November 1991 group actually gave birth 129-181 days after being isolated, while pregnancies in the October 1993 group lasted for 116 229 days after isolation [4]. This suggested that significant delays had been triggered during many of the pregnancies. The possibility was dismissed that the much-delayed births might have been attributable to prolonged sperm storage in the females, because no evidence for this was observed in previous work with captive-bred Carollia $[16,17]$. This has since been confirmed by further studies $[18,19]$.

When pregnancies were then established by timed matings during their first year in captivity, a remarkable range of gestation periods (105 - 178 days; mean $145 \pm 19$ days SD) was again noted. When Carollia were bred during their second year in captivity, there was still a considerable range of gestation periods, but the spread was reduced ( $113-169$ days; mean $127 \pm 12$ days). These data were all for pregnancies that culminated in live births and successful rearing of the young [4].

These results suggested that gestation length in captive-bred Carollia might be influenced by female age, parity or stress. To test these possibilities, a group of nonparous females that had been born and raised in captivity were bred. Unfortunately, this experiment was disrupted by serious malfunctioning of the heating and ventilation systems in the animal facilities and the need to install air conditioning equipment in the bat room. This may have been responsible for more miscarriages in the experimental group and a broad range of gestation periods for pregnancies carried successfully to term (113 - 141 days; mean $126 \pm 9$ days).

When this experiment was repeated in the absence of such stress, the range of gestation periods exhibited was still substantial (113 - 159 days; mean $119 \pm 9$ days); however, most $(n=35 / 43)$ fell in the range of $113-119$ days, with another 5 between $123-126$ days. Interestingly, the three longest gestation periods ( 136 - 159 days) were observed in one particular cage. It is unclear if this was attributable to behaviour of the male housed in that cage or its location close to the animal room sink. Some routine husbandry procedures were carried out around the sink (e.g., the cleaning and refilling of food dishes and water dispensers), and these may have been disturbing to the animals. Thereafter, these activities were conducted outside of the animal room. Based upon the results of this experiment, it was concluded that the normal (nondelayed) gestation period for Carollia was $113-119$ days [4]. Additional data subsequently indicated that this should be extended to 113 - 120 days [JJ Rasweiler IV \& NK Badwaik, unpubl. observ.].

These observations indicate that wild-caught Carollia in particular remain sensitive to stress, even after prolonged periods in captivity, and this can significantly lengthen the duration of gestation. This occurred despite the animals having been maintained in limited-access quarters, with continued efforts being made to minimize exposure to potentially stressful stimuli. This indicates that only captive-reared Carollia should be used for studies of nondelayed pregnancies. This actually is not a problem, because Carollia readily rears young in captivity, and the females must be regularly bred in order to preserve their reproductive health $[10,20]$.

This also means that care must be exercised in the design and interpretation of experiments with Carollia and related species seeking to elucidate the physiological mechanisms responsible for postimplantational periods of diapause. For example, previous efforts to alter the seasonal embryonic diapause in the closely related Macrotus californicus [21, 22] may have been affected by the stress associated with maintaining the animals in captivity. 


\section{Effects of experimental food deprivation upon gestation length}

When Carollia are fed every night in captivity, they are homeothermic. When food-deprived, however, they will drop their body temperature and go into diurnal torpor during the next day. Particularly in the wild, this is accompanied by pronounced behavioural changes, such as entrance into a state of deep lethargy and a greater propensity to cluster closely together. The state of torpor persists until the animals spontaneously arouse and have access to food on the following evening [23].

When captive-reared and -bred Carollia were experimentally food-deprived one night per week between days 5 and 100 post coitum (p.c.) in warm (24 - 26 C) animal quarters, the following gestation periods were recorded: 2 bats -114 and 116 days; 22 bats -122 to 163 days (with 13 pregnancies being $>130$ days); and 2 bats -201 and 237 days. The mean gestation period was 143 \pm 26 days SD. Thus, only two bats had gestation periods within the normal range for captivereared animals (113 - 120 days), and the prolongation of pregnancy in the remaining females must have been attributable to developmental delays [24]. These animals were all sexually-isolated on day 5 p.c., and no evidence of prolonged sperm storage has been observed in other studies with the same colony $[18,19]$. Because the animal quarters were warm and food-deprivation was limited to one night per week, it seems likely that the most lengthy delays were triggered by stress rather than limited periods of modest hypothermia. It is also noteworthy that the periods of delay differed so markedly between animals that were all treated similarly.

\section{Seasonal embryonic diapause in Carollia perspicillata}

The prolonged lengthening of gestation observed after wild-caught Carollia were sexually-isolated in captivity during October or November, plus preliminary observations on birth periods in the wild, suggested that animals in the latter population might exhibit a seasonal embryonic diapause. To investigate this, 20 females of adult body size were collected from a wild colony on Trinidad on November 9, 1995 and their reproductive tracts were examined histologically. The minimum postcoital ages of the embryos they carried were estimated by comparisons with those conceived after timed, captive matings $[12,16]$. Then, another 20 adult females in advanced pregnancy or carrying neonatal young were collected from the same colony on April 1, 1996 to establish the approximate timing of the next birth peak [4].

One nonpregnant animal collected in early November had growing, vesicular follicles in her ovaries and exhibited early preovulatory stimulation of her oviducts. All of the remaining 19 females were in early pregnancy. Each carried a single embryo at the following stages: 1 pronuclear ovum (estimated age day 2 p.c.); 1 oviductal morula (day 9 p.c.); 4 implanting blastocysts (days $16-17$ p.c.); 4 implanted blastocysts (days $19-22$ p.c.); and 9 primitive streak embryos (days $28-34$ p.c.).

It seems likely that the ages of at least some of the more advanced embryos may have been underestimated. Small amounts of highly invasive trophoblast were observed in the myometria of one uterus carrying an implanted blastocyst undergoing amniogenesis, and 4 uteri bearing primitive streak stage embryos. Such trophoblast was moderately to extremely abundant in the myometria and sometimes adjacent extrauterine tissues of the remaining 5 uteri bearing primitive streak stage embryos. Such trophoblast is regularly seen in delayed pregnancies of Carollia, but not in normal ones (see below) [4, 25].

Most of the females collected on April 1, 1996 carried near-term fetuses ( $n=6 / 7$ pregnant animals) or recently-born infants ( $n=13 / 13$ females). This was determined by comparing their 
masses to those of 12 infants born in the captive colony on their day of birth [4] or with a postnatal growth curve for captive born Carollia of Trinidadian descent [14].

Using conservative estimates for the time of conception in females carrying embryos in the wild colony in early November and the likelihood that the middle of the subsequent parturition period for the same colony was about April 1, total gestation periods were calculated to be approximately 163 days. Given that the normal gestation period for Carollia ranges between $113-120$ days, pregnancies during this time of year in the wild would appear to include a minimum diapause of about $44-50$ days. As some of the females captured during November seem to have already taken their pregnancies into diapause, however, actual gestation lengths may have been longerpossibly closer to 180 days or more in length.

\section{Onset and termination of the postimplantational delay}

Most (if not all) of the diapause in Carollia occurs at the primitive streak stage. When wild-caught, captive-bred animals were examined between days $28-60$ p.c., considerable variation was noted in development of their embryos, even when the specimens were processed on the same postcoital day. Embryos were frequently found at the primitive streak stage between days $28-50$ p.c., and even as late as day 60 p.c. in one case. This was presumably because of stress-induced delays in many of those females. In other females examined between days $34-60$ p.c., however, their embryos had progressed to the somite, limb bud or even later stages. There was no evidence of embryos being held for a prolonged period at or after the somite stage. The maximum uterine diameter (reflective of the contained products of conception) then began to increase steadily with post-coital age, and the somite stage was associated with important morphogenetic changes in the placenta (see below) [4, 25].

Similarly, when embryos reach the somite and limb bud stages in the wild - generally in January on Trinidad - it indicates that the diapause is over, and their development then progresses steadily to term. In other words, development then seems to progress in approximate temporal agreement with what occurs in normal (nondelayed) pregnancies in captive-bred animals $[10,26]$.

\section{Annual reproductive cycle of Carollia in the wild}

Abundant evidence has now been accumulated that most adult female Carollia bear two young per year on Trinidad during roughly synchronized reproductive periods. Their first pregnancy appears to be established between September and November, includes a period of embryonic diapause, and is completed in March or April. Furthermore, embryos collected and staged from females in the wild population during late January for 8 years, generally revealed that many pregnancies had recently exited diapause at that time. After parturition, most parous females then apparently quickly conceive again at a postpartum estrus [10, JJ Rasweiler IV, CJ Cretekos \& RR Behringer, unpubl. observ.]. In captive females, a postpartum estrus usually occurs between $3-6$ days after parturition, but may sometimes be several days later [27].

The second pregnancy apparently does not include a significant period of diapause. When many parous females were sampled in late May on Trinidad during 17 different years, most carried conceptuses that had progressed to the somite stage or beyond [10, 27; JJ Rasweiler IV, CJ Cretekos \& RR Behringer, unpubl. observ.]. Based upon comparisons to timed pregnancies in captive-bred animals [26], this is what would be expected if the field pregnancies had been established during March or April and were of normal length. 


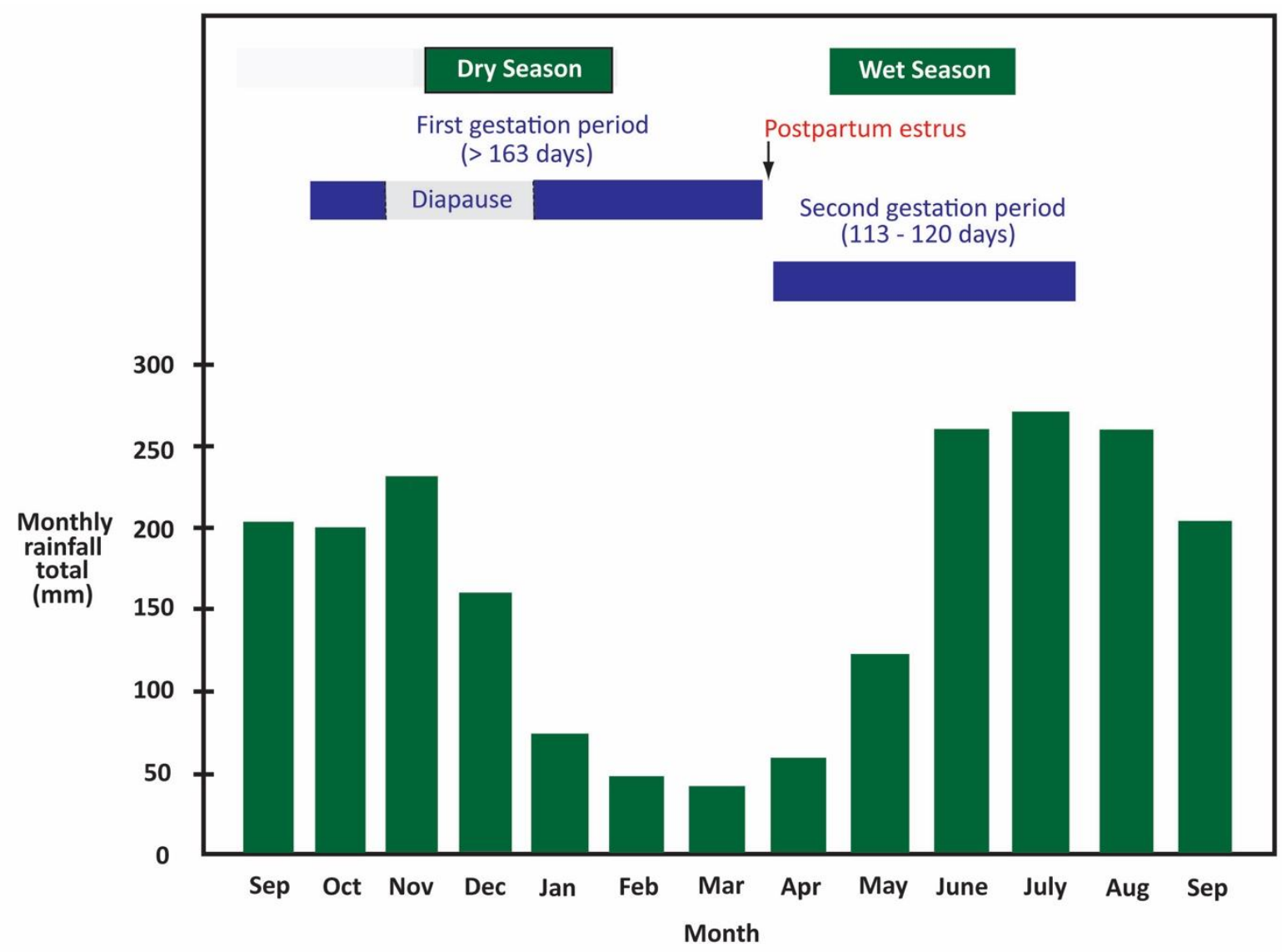

Figure 1. The annual reproductive cycle for female Carollia perspicillata collected in central to eastern Trinidad. This is plotted along with average monthly rainfall totals recorded over a 30 year period in the same region by Meteorological Services, Piarco International Airport, Trinidad, West Indies. Most adult females carry two pregnancies each year. The first one, ending in the dry season, includes a period of diapause. The second one, conceived at a postpartum estrus and ending in the wet season, lacks a diapause and is normal in length.

The adaptive significance of a seasonal diapause during one of Carollia's two synchronized reproductive periods in the wild remains speculative. This diapause is approximately two months long, and its occurrence ensures that most births occur during the second, rather than the first, half of Trinidad's long dry season (Fig. 1). This dry season is sometimes, but not always, harsh. Trinidad's rainy and dry seasons are also variable in their onset and termination [28; JJ Rasweiler IV \& NK Badwaik, pers. observ.] For reasons yet to be determined, a birth peak around April 1st may be a more advantageous time for females to meet the nutritional demands of advanced pregnancy, lactation, and the quick establishment of a second pregnancy while simultaneously lactating. This would also be a period when the rapidly growing first infant has substantial nutritional requirements.

Although Carollia exhibits a postpartum estrus soon after parturition in captivity and at least one of its annual pregnancies in the wild (i.e., the pregnancy including the diapause), no evidence has been obtained of a lactation-associated diapause. Most females bred at a postpartum estrus in captivity experienced gestation periods of normal or near-normal length [4]. This also generally appears to be the case for pregnancies similarly conceived in the wild around the April birth peak. This is perhaps not surprising, because early development in Carollia is normally very slow, with the embryos only reaching the primitive streak stage by the end of their first month [12]. As the gravid uterus at this stage has barely begun to swell [25], early pregnancy should not be a physiological burden for females simultaneously lactating. 
Thus, in contrast to most species demonstrating an embryonic diapause [1], Carollia exhibits neither a facultative (lactational) diapause nor an obligate diapause in every pregnancy. Rather, pregnancies during one synchronized reproductive period include a diapause of about 2 months, whereas those in the immediately following reproductive period do not. An Indian megachiropteran bat, Cynopterus sphinx, exhibits a similar dichotomy during its two pregnancies each year $[6,29]$.

The environmental cue that keeps the annual reproductive cycle of female Carollia relatively synchronized in the wild is not known. Although delays in the wild could conceivably be triggered by stress, but of a seasonally-recurring nature, it is difficult to imagine what this might be. On Trinidad embryos carried by these bats go into a diapause late in the wet season. Because Carollia is a food generalist (feeding on fruits, nectar/pollen and insects), it seems unlikely that food availability should be a problem at that time of the year [30-33]. Rather, it appears that these bats have evolved an annual cycle that anticipates when advanced gestation, lactation, rapid neonatal growth, and the initiation of new pregnancies might be optimally timed some months into the future, after completion of the diapause. Presumably this occurs for nutritional reasons. It is also noteworthy that, although Carollia were experimentally subjected to substantial stress in some of our work with captive animals, the delays induced were highly variable in duration (i.e., from a few days to several months). This suggests that environmental stress might be a highly unreliable cue for triggering periods of diapause of appropriate length in the wild.

Interestingly, the timing of birth peaks for the Carollia population living in French Guiana relative to the rainy and dry seasons [11] are quite similar to those on Trinidad, although the occurrence of a period of diapause in the former population needs to be confirmed. The French Guianese animals were collected at about $5^{\circ} \mathrm{N}$ latitude, where the maximum annual change in daylength is only about 35 minutes. On Trinidad, at $10-11^{\circ} \mathrm{N}$ latitude, this is approximately 73 minutes [9].

\section{Why diapause in Carollia is postimplantational}

In most mammals exhibiting an embryonic diapause, this occurs at the preimplantation blastocyst stage. It has long been recognized that some bats are unusual in deferring the diapause until after implantation, but the reasons for doing so have remained an enigma. Diapause has two important functions; in some species it provides a mechanism to delay parturition until a time of year which is environmentally favorable for offspring survival; in others it delays the development of embryos in females that are still nursing the young from a previous pregnancy [2]. One might conclude that pre- and postimplantational diapause are simply alternative solutions that evolved to address these challenges.

Now that Carollia has been studied quite thoroughly, it is clear that its postimplantational diapause is associated with a variety of other unusual reproductive specializations. All seem to have evolved to promote optimal vascularization of the placenta. This, in turn, permits the production of highly precocious young which, given the natural history of these animals, probably enhances their survival. One must question whether having a preimplantational diapause, with a prolonged free-floating blastocyst stage, would permit Carollia and related species to accomplish these goals as successfully. These specializations are as follows:

Site of implantation and positioning of the definitive placenta. Carollia has a uterus which is externally simplex. If substantial postovulatory development of the endometrium has occurred, the uterus has two short internal cornua with endometrial glands interposed between the uterotubal junction and the main uterine cavity (Fig. 2). These have been termed "intramural 
uterine cornua" (IUCS). During pregnancy, the single blastocyst attaches in the IUC on the side of ovulation or at its mouth. When attachment occurs at the distal end of the preferred implantation zone, trophoblast invasiveness and decidual overgrowth of the blastocyst still ensure successful implantation in an optimal location. The blastocyst does not enter and float freely in the uterine cavity for any significant period.

Initially, the implantation site is vascularized largely by branches of the uteroovarian artery and vein ipsilateral to the ovary containing the $\mathrm{CL}$. As the blastocyst grows, however, preferential trophoblastic growth shifts the embryo and its horseshoe-shaped preplacenta (chorionic placenta) preferentially towards the uterine midline. This permits the developing placenta to also tap major blood vessels on the contralateral side of the uterus.

As pregnancy advances, the now chorioallantoic placenta becomes progressively more discoidal in shape and centered on the fundus of the uterus. The placenta always develops at exactly the same location in the uterus, and those examined in advanced pregnancy appear to be equally vascularized by maternal vessels approaching from both sides of the uterus (Fig. 3). This dual vascularization permits the females to produce very large and precocious infants. These weigh 26.9 to 36.9 per cent of the mean mass of recently mated adult females, have open eyes, and a well-furred dorsal surface [34].

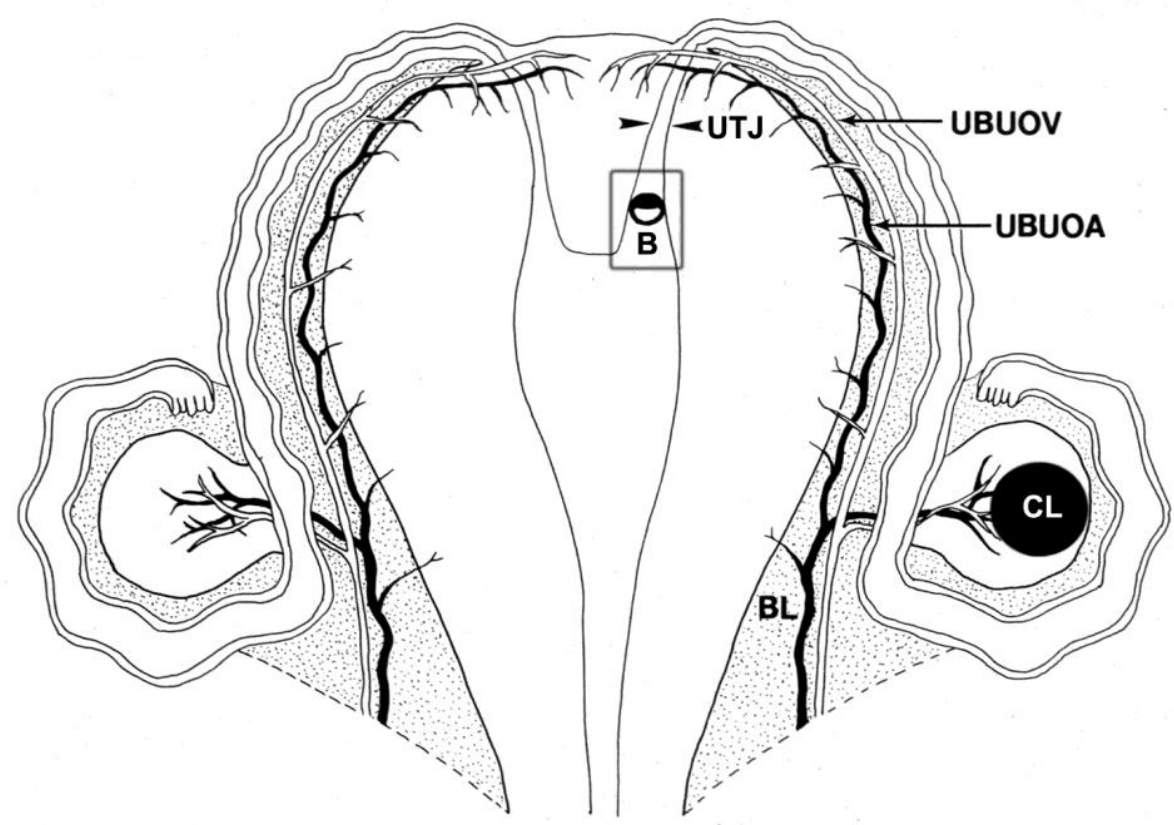

Figure 2. Diagrammatic representation of the reproductive tract of Carollia in frontal section. Initial attachment and implantation of the blastocyst $(B)$ occurs within the zone depicted by the rectangle. This lies within, or at the mouth of, the intramural uterine cornu between the uterotubal junction (UTJ) and the main uterine cavity ipsilateral to the ovary containing the corpus luteum $(\mathrm{CL})$. $\mathrm{BL}=$ the main blood supply to that side of the tract. UBUOA and UBUOV = uterine branches of the uteroovarian artery and vein. Modified and reproduced with permission from [34]. 


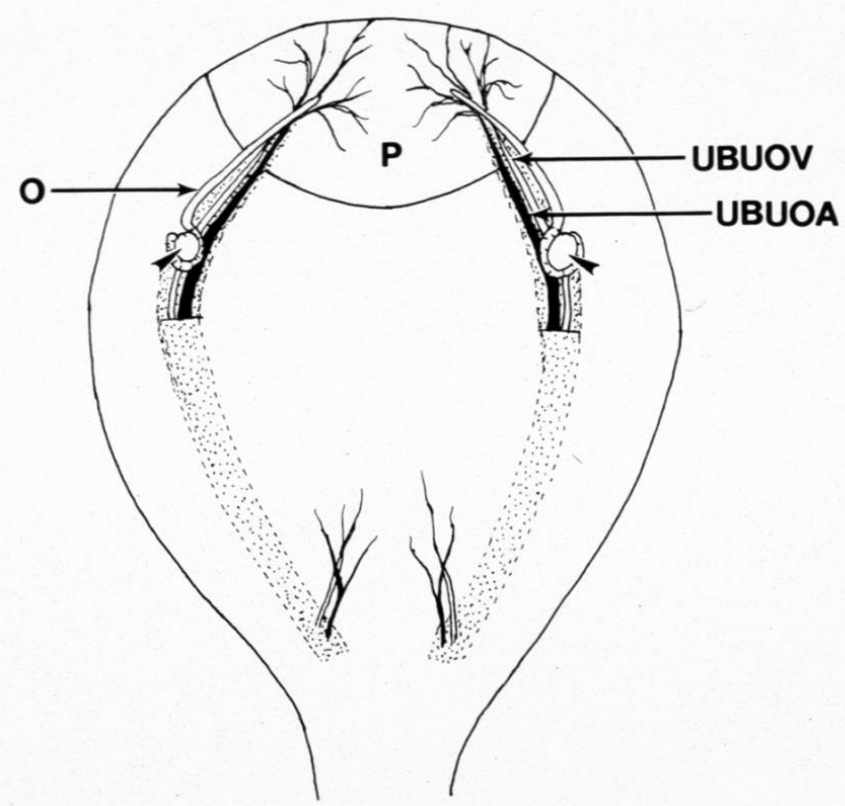

Figure 3. Diagramatic view of the dorsal surface of the uterus from a Carollia in advanced pregnancy. The discoidal chorioallantoic placenta $(P)$ has become centered on the uterine fundus and vascularized equally by the uterine branches of the uteroovarian artery (UBUOA) and vein (UBUOV) on both sides of the tract. The arrowheads denote the ovaries. $\mathrm{O}=$ one of the oviducts. Reproduced with permission from [34].

Development to this state at birth is undoubtedly important, because the baby must possess considerable muscular strength and motor skills to attach to one of its mother's teats and adhere firmly to her while she roosts (suspended by her feet) or flies. Mothers fly with the babies attached for a couple of weeks after birth. Continued growth must then make it necessary for the infant to hang by itself, while its mother forages. To fall off the mother would potentially subject the infant to direct injury and a variety of predators. Unlike many other small mammals, bats do not build nests that would serve to contain and protect altricial young [34].

Preimplantation development of the embryo. Preimplantation development of the embryo is unusual in Carollia and apparently evolved to facilitate implantation within the fundic endometrium. The tubal journey of the embryo takes $11-13$ days, which is one of the longest observed in mammals [35]. Furthermore, the embryo develops to the blastocyst stage, sheds its zona pellucida (ZP), and undergoes significant expansion all within the oviduct $[12,36]$. In most other mammals, embryos enter the uterus at earlier stages, after more rapid transport through the oviducts. The oviduct also appears to have assumed greater responsibility for sustaining the preimplantation embryo in Carollia. During much of the embryo's tubal journey, the oviductal secretory cells appear engorged with glycogen and some lipid droplets [35, 37]. All of these specializations seem designed to prepare the blastocyst for attachment very soon after entry into the uterus.

Differential transport of embryo, unfertilized ova and discarded zonae pellucidae. In Glossophaga soricina, which belongs to the same family as Carollia, normally only living embryos are transported into the uterus. This also occurs at the blastocyst stage and after shedding of the ZP. Unfertilized eggs and discarded ZPs are generally retained in the oviducts, where they may persist through several nonpregnant cycles and even be passed by a living embryo in the event of a successful conception [37, 38].

This selective transport process may have evolved to prevent the passage of unfertilized eggs or ZPs into the uterus, where they might interfere with initial attachment of the blastocyst. The 
process of blastocyst implantation in G. soricina is very similar to what has been described for Carollia, i.e. usually within the restricted confines of an IUC [39]. The remnants of unfertilized eggs have not been seen in the oviducts of Carollia, and discarded ZPs were rarely found in the oviducts after days 15-16 p.c. This may be due to the actions of a zona-lysin. Limited observations suggest, however, that normal development may still be required for embryo transport into the uterus [36].

Early uterine luminal closure and blastocystic attachment. Implantation has been studied in Carollia after timed, captive matings, following vascular perfusion fixation and plastic embedding (which minimize shrinkage artifacts), and with the electron microscope. These studies have revealed that uterine luminal closure usually occurs prior to, or at the time of, transport of the blastocyst to the usual implantation site (Fig. 4). Also, trophoblastic apposition and adhesion to the uterine epithelium are initiated very soon after entry of the blastocyst into the uterus. This minimizes the "free-floating" period of the blastocyst in the uterus, and in no case has a blastocyst been observed that had passed beyond the normal implantation zone [36]. This differs considerably from species like the mouse in which embryos are transported into the uterus, spaced along its length, lose their ZPs, and closure is then initiated immediately before implantation [40].

It has generally been considered that the transport of blastocysts to their implantation sites is accomplished by myometrial contractions [41, 42]. In the case of Carollia, it deserves to be asked if the oviductal musculature may have assumed greater responsibility in the process. That is because of the short distance between the uterotubal junction and the implantation site, the paucity of fluid around free uterine blastocysts (Figs. 2,4), the disposition of smooth muscle in Carollia's simplex uterus, and the failure to ever see unattached blastocysts distal to the usual implantation zone [36]. Also, the intramural UTJ is notable for having a rich content of elastic fibers which presumably restricts its patency [18].

\section{Unique uterotubal orientation of the inner cell mass at the time of initial blastocystic} attachment. In most eutherian mammals, at the time of first attachment of the blastocyst to the endometrium, its inner cell mass (ICM) exhibits a species' specific orientation relative to the uterus. Typically the ICM faces towards the uterine mesentery (mesometrial), away from the mesentery (antimesometrial), or in an intermediate direction (lateral or orthomesometrial). This is usually correlated with subsequent development of the fetal membranes and definitive chorioallantoic placenta. For example, in many mammals the first chorioallantoic placentation is established dorsal to the embryo [41-43]. Carollia and some other bats exhibit a seemingly unique orientation, i.e., towards the uterotubal junction (Figs. 2,5 ). This may be more conducive to subsequent development of a discoidal, chorioallantoic placenta that is centered on the uterine fundus and equally vascularized by branches of the uteroovarian vessels on both sides of the reproductive tract [35]. This, in turn, presumably provides essential support for the development of a larger, more precocious infant. 


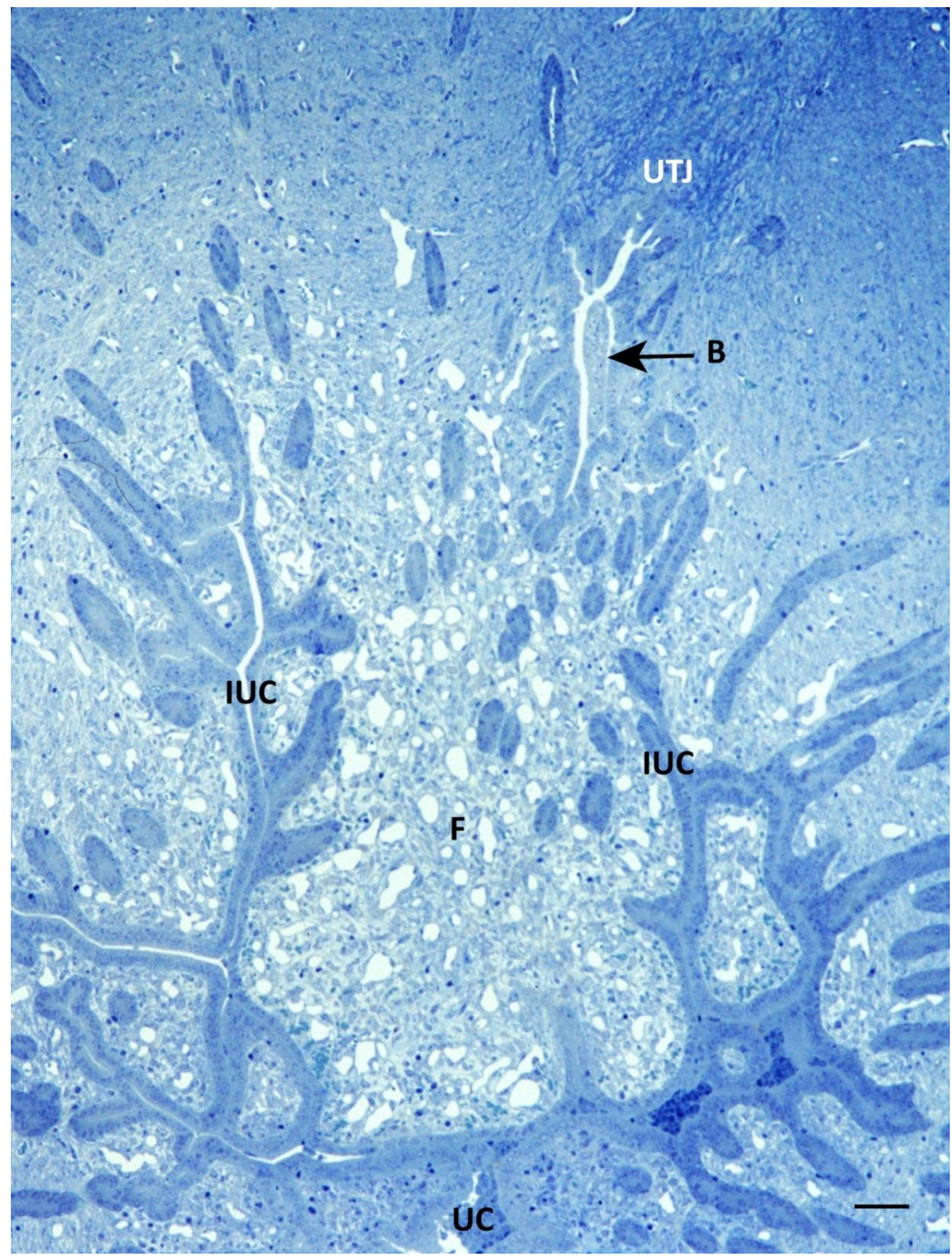

Figure 4. Frontal section of a perfusion-fixed, plastic-embedded uterus bearing a partially collapsed blastocyst (B). This was obtained from a captive-bred Carollia on day 12 p.c. The blastocyst was located in one of the intramural uterine cornua (IUCS), very close to the uterotubal junction (UTJ). Although the blastocyst was not yet within the normal implantation zone (further down that IUC), luminal closure was nearly complete in both IUCS and the main uterine cavity (UC). Note that the fundic endometrium (around F) appeared more edematous, with more dilated capillaries, than the remaining endometrium. Toluidine blue. Bar $=50 \mu \mathrm{m}$. 


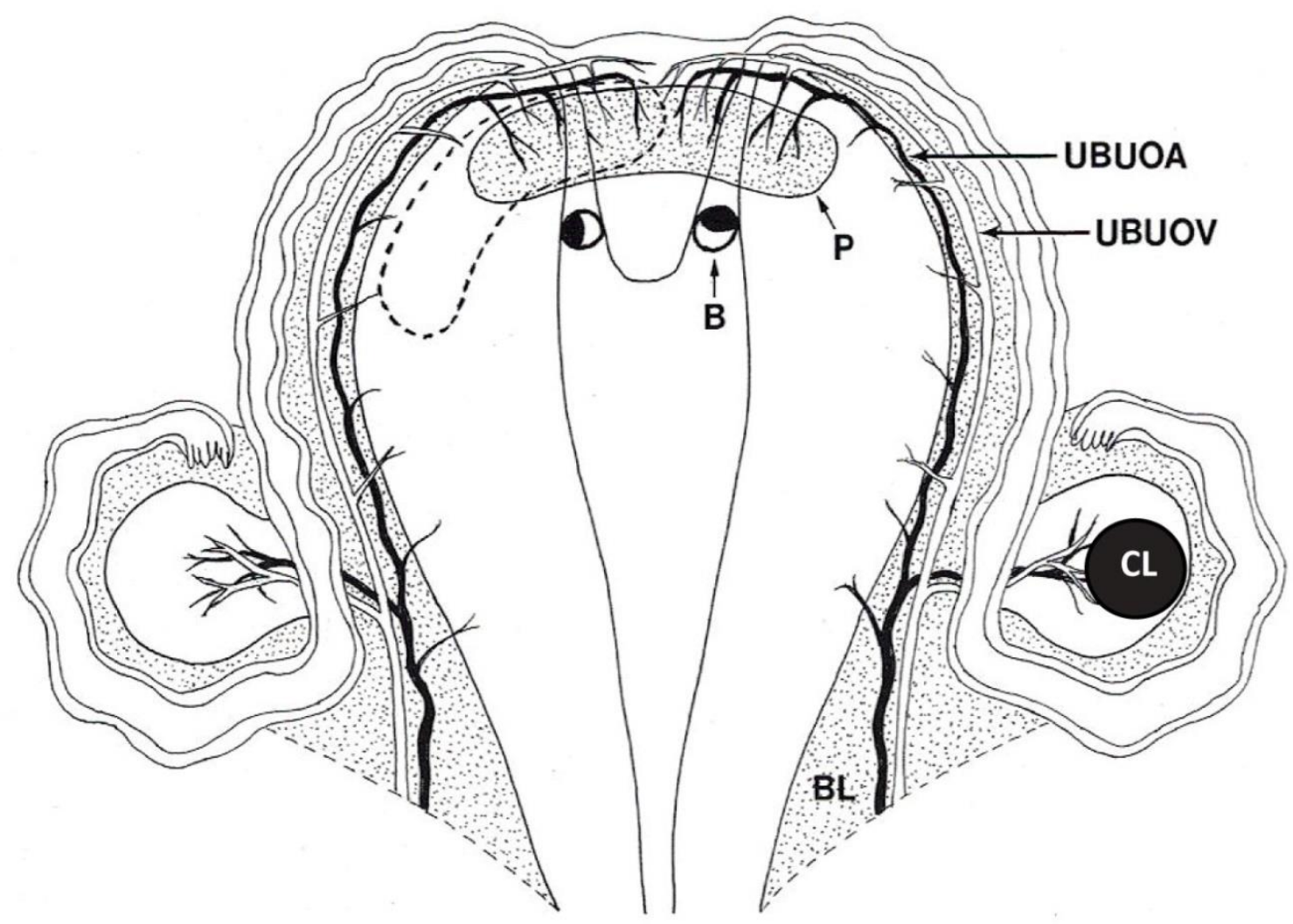

Figure 5. Diagramatic representation of the reproductive tract of Carollia depicting two possible orientations of the inner cell mass of the blastocyst (B) upon initial attachment to the endometrium. The blastocyst on the right depicts correct orientation of the inner cell mass towards the uterotubal junction. This is presumably more conducive to development of the definitive placenta $(P)$ on the midline of the fundus and its equal vascularization by major blood vessels $(\mathrm{BL})$ serving both sides of the uterus. $\mathrm{CL}=$ corpus luteum. $\mathrm{UBUOA}=$ uterine branch of the uteroovarian artery. UBUOV = uterine branch of the uteroovarian vein. Modified and reproduced with permission from [35].

Preferential ovarian stimulation of one side of the female reproductive tract. A variety of bats have been found to exhibit preferential stimulation of the female reproductive ducts ipsilateral to the ovary containing the preovulatory follicle or new corpus luteum. In the case of Carollia, which has a simplex uterus, such differential stimulation has only been observed to affect the oviducts. In some bats with bicornuate or duplex uteri, however, this also extends to the uterus/uteri. The condition of their oviducts has not always been characterized.

For many years, since first observed in the giant flying fox (Pteropus giganteus, a megachiropteran fruit bat) $[44,45]$, this was a puzzle. It has generally been believed by reproductive physiologists that most steroids produced by the ovaries enter the systemic circulation and are then delivered to the reproductive ducts on either side at equal levels. Where unilateral stimulation occurs, there must be either a local route for the delivery of these hormones and/or differential responsiveness of the ducts on either side.

When such unilateral stimulation was observed in Carollia and a closely related bat, Noctilio albiventris, it was suggested that this might be primarily due to a countercurrent exchange of hormones between the ovarian venous and/or lymphatic drainage and the arterial supply of the ipsilateral reproductive ducts. The vascular anatomy of their reproductive tracts is consistent with this possibility $[16,46,47]$. Much better evidence for the occurrence of a local counter- or crosscurrent transfer of ovarian steroids was subsequently obtained through elegant studies of 
three species of Australian Pteropus. These established that 1) a branch of the uteroovarian artery vascularizes the cranial end of the ipsilateral uterus in these bats, 2) the anatomical relationship between ovarian venous and arterial vessels is unusually intimate in the presumed region of steroid transfer, and 3$)^{3} \mathrm{H}$-estradiol microinjected into the ovary of one species was preferentially transported into the ipsilateral uterus $[48,49]$.

In the megachiropterans that have been adequately studied, implantation generally occurs at the cranial end of the uterus ipsilateral to the ovary containing the new corpus luteum and, at least in some, this region exhibits preferential stimulation prior to implantation $(6,35,44,45,48-54]$. In two species, this seems to also be associated with early closure of the uterine lumen. This may prevent transport of the embryo further down the uterus, instead restricting it to the most favourable site for placental development $[52,54]$. The unilateral endometrial reaction occurring in a microchiropteran bat, Peropteryx kappleri, seems to have a similar effect upon embryo transport [55]. The timing of implantation relative to embryo transport into the uterus is unknown for these species, but at least in Rousettus spp. development progresses to the blastocyst stage in the oviduct [35]. This may also promote embryo attachment relatively soon after its passage into the preferentially-stimulated portion of the uterus.

In most species of megachiropterans found to exhibit a diapause, implantation occurs within the preferentially-stimulated portion of the uterus, and the diapause is postimplantational $[6,52,54]$. Presumably, by avoiding a prolonged free-floating blastocyst stage, the chances of attachment outside of that zone are minimized. There is one megachiropteran (Eidolon helvum) that was instead reported to exhibit a preimplantation diapause [56]. The embryo pictured in that study had actually implanted, however, and was associated with a well-developed preplacenta. As the yolk sac had shrunken during histological processing, it was mistakenly interpreted to be a freefloating blastocyst.

In the case of Carollia, it is impossible to identify an advantage to differentially stimulating just the two oviducts. This does not seem energetically consequential, as these small organs only exhibit such stimulation immediately around ovulation and, in most adult females in the wild, this is limited to twice a year. This is most likely a primitive reproductive characteristic retained from ancestral bats with duplex or bicornuate uteri that also locally stimulated the optimal implantation and placentation site in their uterus.

This is strongly supported by comparative studies of Carollia and a closely-related bat, $N$. albiventris. In contrast to Carollia, N. albiventris has a partially bicornuate uterus and exhibits differential stimulation of both the oviducts and uterine cornua prior to implantation. On the other hand, like Carollia, N. albiventris exhibits development to the ZP- free blastocyst stage in the oviduct, possibly the differential transport of living versus dead ova, and blastocyst implantation at the cranial end of the uterine cornu ipsilateral to the $C L$ (i.e., at an implantation site homologous to that of Carollia) [34, 46, 57].

\section{Preferential growth of trophoblast towards the uterine midline after implantation. Although} initial attachment of the blastocyst in Carollia is usually circumferential in the IUC on the side of ovulation, its trophoblast then grows preferentially towards the uterine midline. This results in the discoidal placenta eventually becoming perfectly centered on the fundus and equally vascularized by the main uteroovarian vessels on both sides of the tract (Figs. 2, 3)[13, 34].

In order for this to occur, there must be something different about the fundic endometrium to preferentially attract the trophoblast. There are indeed differences in the endometrium, and some of these were seen advantageously in plastic thick sections of uteri that had been perfusion fixed. In several specimens that carried oviductal blastocysts or a uterine blastocyst that had not yet entered the normal implantation zone, more capillaries in the fundic endometrium appeared 
dilated, and its stroma more edematous (Fig. 4). These differences in the fundic endometrium became even more pronounced as implantation progressed (Fig. 6). As vigorous trophoblastic invasion occurs circumferentially around the blastocyst, the endometrium surrounding the IUC at the implantation site is penetrated. Thus, the pronounced reaction of the fundic endometrium cannot be attributed to trophoblastic invasion only towards the uterine midline.

Throughout the preimplantation period, there was also a variable degree of extravasation of erythrocytes into the endometrial stroma. This was most common in the fundic endometrium and area immediately around the IUCs (Fig. 7). None of the observed vascular leakage appeared to be artefactual, because it was also seen in immersion-fixed, paraffin embedded specimens, and the phagocytosis of erythrocytes by endometrial fibroblasts and macrophages was observed with the electron microscope $[12,34,36]$. This was so common and pronounced that the extravasated blood marked early implantation sites and eliminated the need to inject Pontamine sky blue for that purpose.

These observations indicate that the fundic endometrium of Carollia is a unique part of the uterus. Which of its components may be promoting trophoblastic growth remain unclear, but at least some suspicion must fall upon the endothelial cells of its capillary beds. In another bat, Molossus rufus (also widely referred to as Molossus ater), the definitive chorioallantoic placenta always forms in exactly the same uterine location, i.e., interior to the uterotubal junction in the right uterine cornu. This positioning is determined by a morphologically distinct population of maternal endometrial endothelial cells that develop in the region, stimulate local trophoblastic proliferation, and provide a scaffold for trophoblastic growth during early placental development $[58,59]$.

In summary, all of these reproductive specializations exhibited by Carollia or closely related bats seem to have evolved to ensure blastocyst implantation at an optimal site for subsequent placental development. There is no evidence in these species that mechanisms exist to transport a blastocyst back to that site from more distal parts of the uterus. Such

mechanisms would presumably have to exist in Carollia, if its diapause was instead preimplantational, and the blastocyst was held for that period in the main uterine cavity.

In humans, another monotocous species, abnormal (lateral) positioning of the placenta is more likely to be associated with intrauterine growth retardation. It was postulated that such sites may receive less total blood flow from the single ipsilateral uterine artery than more central (dorsal or ventral) and preferred regions, which can derive flow from both uterine arteries $[41,60]$. These observations were viewed as evidence that the sites of implantation and resultant placentation in humans may have a significant influence upon pregnancy success .

\section{Placental differences between normal and delayed pregnancies}

To investigate how placentation might be altered during pregnancies including a diapause, reproductive tracts from gravid animals were examined histologically, immunocytochemically, and ultrastructurally. Most of these were obtained after carefully timed, captive matings. By means of comparisons to an embryonic staging system and growth curves for the most rapidly developing pregnancies observed in the captive colony $[4,25]$, the embryos could tentatively be assigned to three groups: 1) normal or near-normal in development; 2) likely in diapause; or 3) likely to have resumed normal development after a period in diapause. These assignments were then confirmed by microscopic examination of the reproductive tracts. Histological studies were also conducted on tracts from 20 females collected in the wild on November 7th, when most had entered or were apparently just entering a period of seasonal diapause [4]. Another three tracts containing 
embryos well into diapause were collected in the wild on December $7^{\text {th }}$ for immunocytochemical analysis [25].

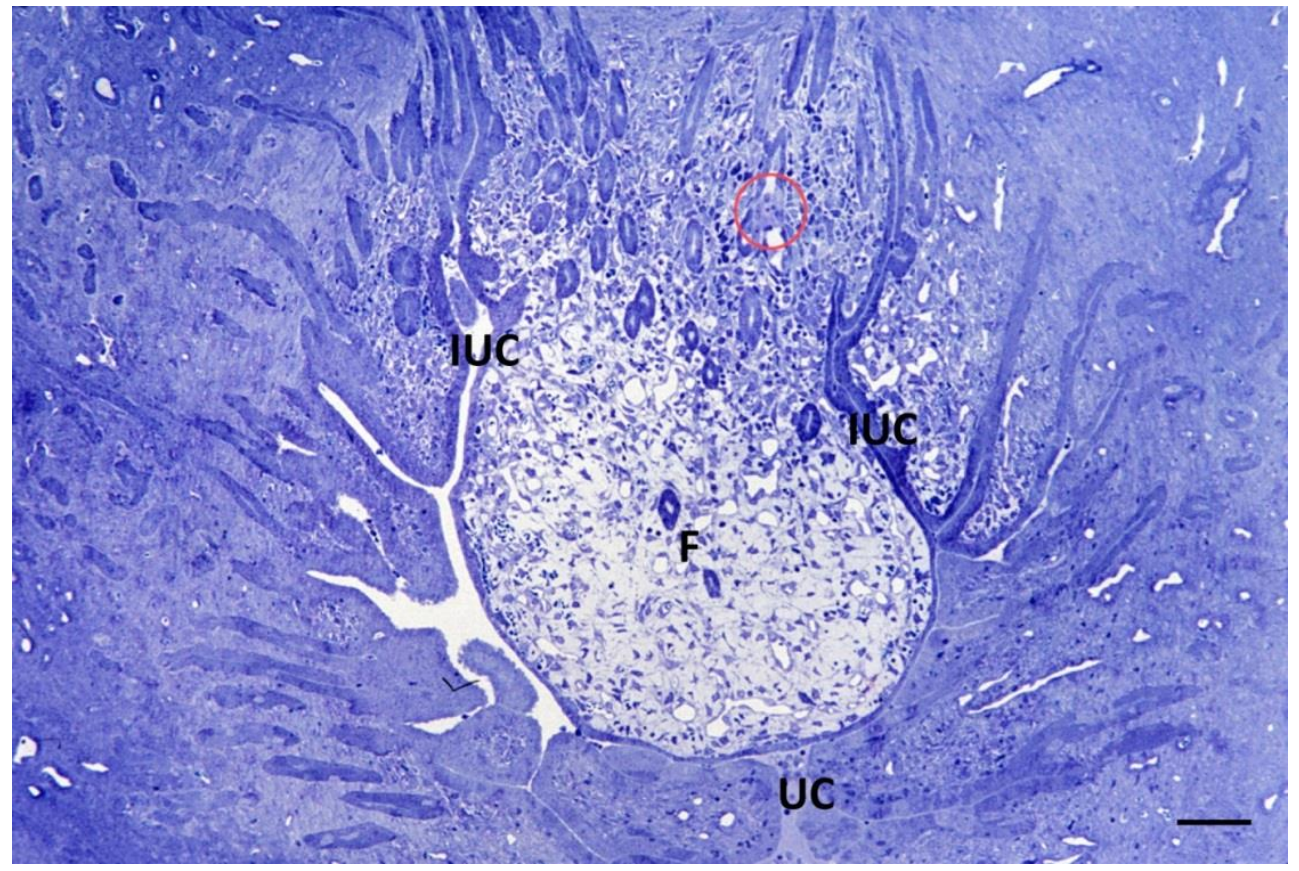

Figure 6. Frontal section of a perfusion-fixed, plastic-embedded uterus carrying an implanting blastocyst. This was obtained from a captive-bred Carollia on day 16 p.c. The blastocyst had begun to implant in the intramural uterine cornu (IUC) on the right, although only a small portion of its trophoblast is evident within the red circled area. The fundic endometrium (around F) appears much more edematous than the remaining endometrium, although that to the right of the IUC had also been invaded by trophoblast. UC = main uterine cavity. Toluidine blue. Bar $=100 \mu \mathrm{m}$.

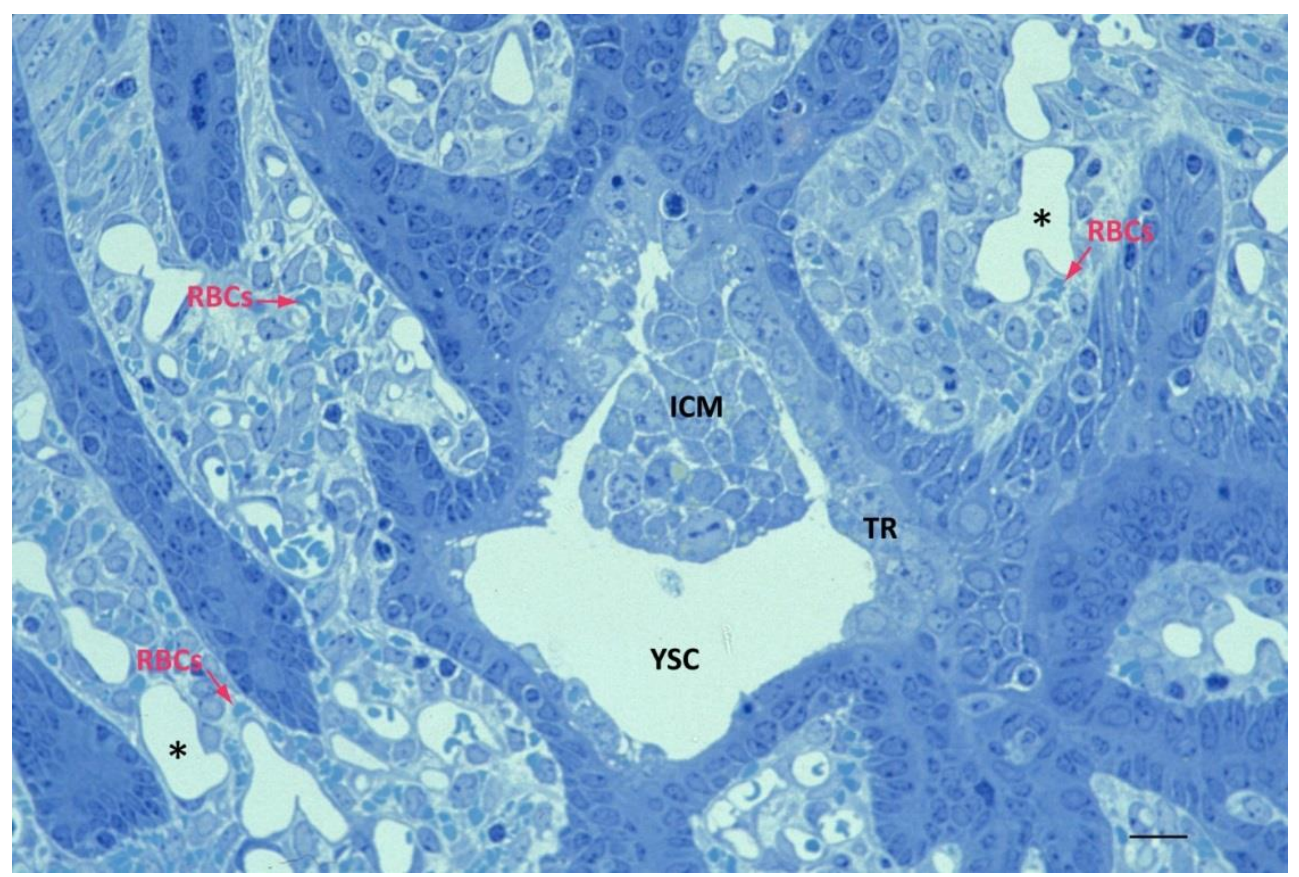

Figure 7. Frontal section of a perfusion-fixed, plastic-embedded uterus bearing an early implanting blastocyst. This was obtained from a captive-bred Carollia on day 13 p.c. The inner cell mass (ICM) was correctly oriented towards the uterotubal junction (not evident in this field). Most of the blood in the capillaries (e.g., at ${ }^{*}$ ) had been flushed out by the perfusion procedure. Abundant erythrocytes (RBCs) are evident, however, in the extracellular matrix of the endometrial stroma. YSC = yolk sac cavity. $T R=$ trophoblast. Toluidine blue. Bar $=25 \mu \mathrm{m}$. 
A distinctive cellular element noted in all tracts carrying embryos in diapause was a population of extraordinarily invasive trophoblast. This was released by the developing placenta and invaded the female reproductive tract via subendothelial routes, primarily along capillaries and venous vessels (Fig. 8). Invasion via an endovascular route was never observed. Because its distribution within the female tracts was highly uneven, its abundance could not be readily quantified; however, this generally seemed to be correlated with the apparent duration of the diapause. Some tracts contained only small amounts, while in others its infiltration of maternal tissues was massive. The depth of invasion was also variable. In some animals it was limited to the myometrium, while in others it extended into the broad ligament, adjacent portions of the oviducts, and sometimes even the ovaries in small amounts [25]. The full extent of trophoblastic invasion could not be determined because, in the most extreme cases, it had progressed to the point where the broad ligaments were clipped during dissection (i.e., between the ovaries and the dorsal body wall).

To confirm the identification of the invasive trophoblast, reproductive tracts were stained immunocytochemically for intermediate filament proteins. Antibodies used included CAM 5.2 and AE1/AE3 (monoclonals directed against a variety of cytokeratins), a mouse monoclonal directed against human leiomyomal desmin, and a mouse monoclonal directed against porcine lens vimentin. The invasive cells were strongly reactive with the antibodies directed against cytokeratins, as would be expected of many epithelial cells. They did not express desmin (which is typical of myogenic cells), but surprisingly they did express vimentin (which is typical of cells of mesenchymal origin). The latter has rarely been seen in the trophoblast of other species, but has been recorded for the highly invasive trophoblast (or its immediate cellular precursors) of several bat species. This can be rationalized, as such invasive trophoblast has the capability to migrate through connective tissues of the reproductive tract $[25,61,62]$. In order to undertake such migrations, cells must undergo an epithelio-mesenchymal transformation, and this is frequently associated with the initiation of vimentin expression [63].

Highly invasive trophoblast was noted in the myometria of all females carrying primitive streak stage embryos collected in early November and December in the wild, at the beginning of or during the seasonal diapause (Fig. 8). Highly invasive trophoblast was never seen in the tracts of captive-bred animals determined to be carrying normal (nondelayed) pregnancies (Fig. 9). It was seen in all captive-bred animals carrying primitive streak embryos in diapause (Fig. 10) or more advanced embryos that were small for their postcoital ages. The latter had probably experienced a period of diapause, and normal development had then resumed [25].

As the diapause progressed, the invasive trophoblast in some Carollia exhibited another highly unusual cytological characteristic; they extended a profusion of cytokeratin- and vimentinpositive, dendritic processes out into their surroundings (Fig. 10). This was seen in some captive females still carrying primitive streak embryos on days 50 and 60 p.c. and in others captured in the wild in early December [62]. An abundance of dendritic, highly invasive trophoblast has also been noted in a closely related bat (Diaemus youngi) late in pregnancy. This may reflect the adoption of a more mesenchymal phenotype by the trophoblast after extended residence in the extracellular matrix of perivascular connective tissues [61]. Similar observations have been made on epithelial cells cultured within three-dimensional gels of type I collagen [64-66].

The functional significance of the highly invasive trophoblast observed during diapause in Carollia is unknown. At least up to day 60 p.c., a comparable population of trophoblast does not develop in normal (non-delayed) pregnancies. In $D$. youngi, however, highly invasive trophoblast vigorously invades the walls of uterine arteries and veins, both within the uterus and its mesenteries. This may play a very important physiological role in $D$. youngi by modifying the tunica muscularis of arteries supplying the uterus to increase blood flow during pregnancy $[25,61]$. 
When the preplacentae of diapause were compared with normal or post-diapause placentae in Carollia, other important differences were noted. The preplacentae of diapause typically had a higher ratio of cytotrophoblast to syncytiotrophoblast (which lines the maternal blood spaces), and had only been invaded to a very limited extent along its interior surface by vascularized extraembryonic mesoderm (Fig. 11). Much of the cytotrophoblast within these placentae was also quite undifferentiated - appearing randomly-oriented (i.e., without obvious apical-basal polarity), being linked only by primitive junctions, and lacking basal laminae. Cytotrophoblast immediately adjacent to the syncytiotrophoblast lining the maternal vascular spaces displayed some apicalbasal polarity (evident in the electron microscope), but also lacked a basal lamina [25, 62].

When embryos had progressed to the somite and limb-bud stages, either directly or after a period of diapause, significant changes became evident in their placentae. At the somite stage the allantois makes first contact with the preplacenta, thereby creating a chorioallantoic placenta. The placenta also then becomes notably larger and thicker, seemingly in good part through the rapid addition of syncytiotrophoblast (Fig. 12). Much of this initially takes the form of trophospongium, i.e., syncytiotrophoblast perforated only by maternal vascular spaces. This must be enlarged by the division and fusion of cytotrophoblast cells in the placenta; the syncytiotrophoblast is nonmitotic. During diapause, differentiation does not progress to trophospongium formation. Highly invasive trophoblast is instead released from the placenta, sometimes in extraordinary amounts $[25,62]$. It deserves to be noted that the somite stage, when the allantois makes initial contact with the preplacenta, also exemplifies a step at which the embryo might be sending important morphogenetic signals to the placental trophoblast.

The trophospongium is noteworthy as well for containing an abundance of rough endoplasmic reticulum. The full significance of this is unclear, but it probably plays a major role in synthesizing and secreting components of the intrasyncytial lamina, a prominent extracellular layer that is engulfed by apical, ectoplasmic processes of the trophospongium and remains as part of the definitive interhemal barrier in Carollia. It has been suggested that the intrasyncytial lamina serves both a morphogenetic function during placental development in many bats and to mechanically strengthen maternal vascular spaces and channels in their chorioallantoic placentae $[62,67]$. Syncytiotrophoblast in the interhemal placental barrier of Carollia is notably deficient in cytokeratin content. A major function of cytokeratins is to help cells resist mechanical stress. Rapid expansion of the trophospongium at the somite and limb bud stages may contribute significantly to a subsequent acceleration in embryonic growth [25]. 


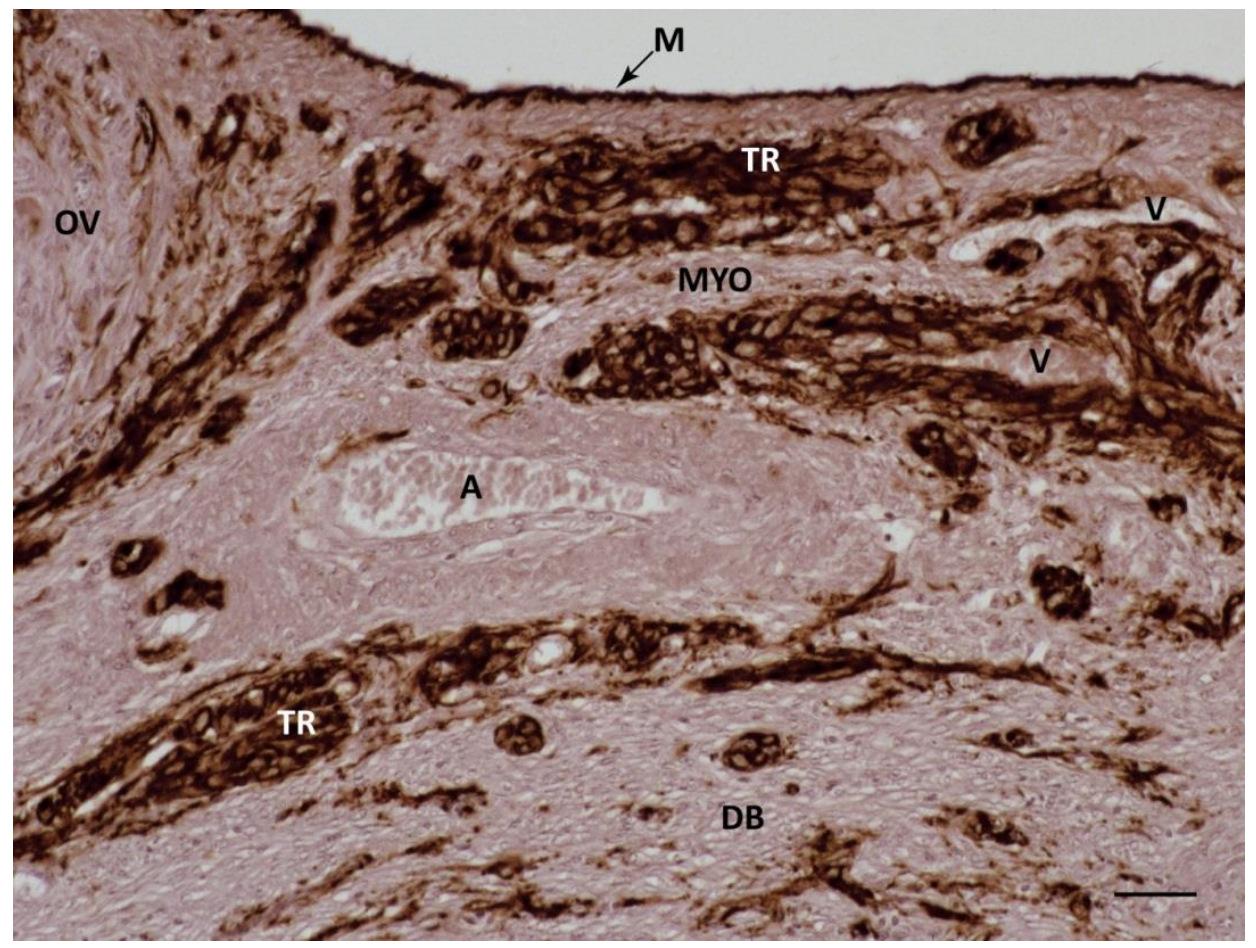

Figure 8. Frontal section of a uterus from a Carollia collected in the wild in early December. The uterus carried a primitive streak stage embryo that had probably been in diapause for a month or more. This section had been stained immunocytochemically with a monoclonal antibody cocktail (AE1/AE3) directed against a variety of cytokeratins. The only reactive elements in this field are abundant, highly invasive trophoblast (TR) primarily in the walls of maternal capillaries and veins (V) and the mesothelium (M). Little trophoblast was associated with a maternal artery $(\mathrm{A}) . \mathrm{DB}=$ decidua basalis. $\mathrm{MYO}=$ myometrium. $\mathrm{OV}=$ tangential section of one oviduct. Bar $=50 \mu \mathrm{m}$.

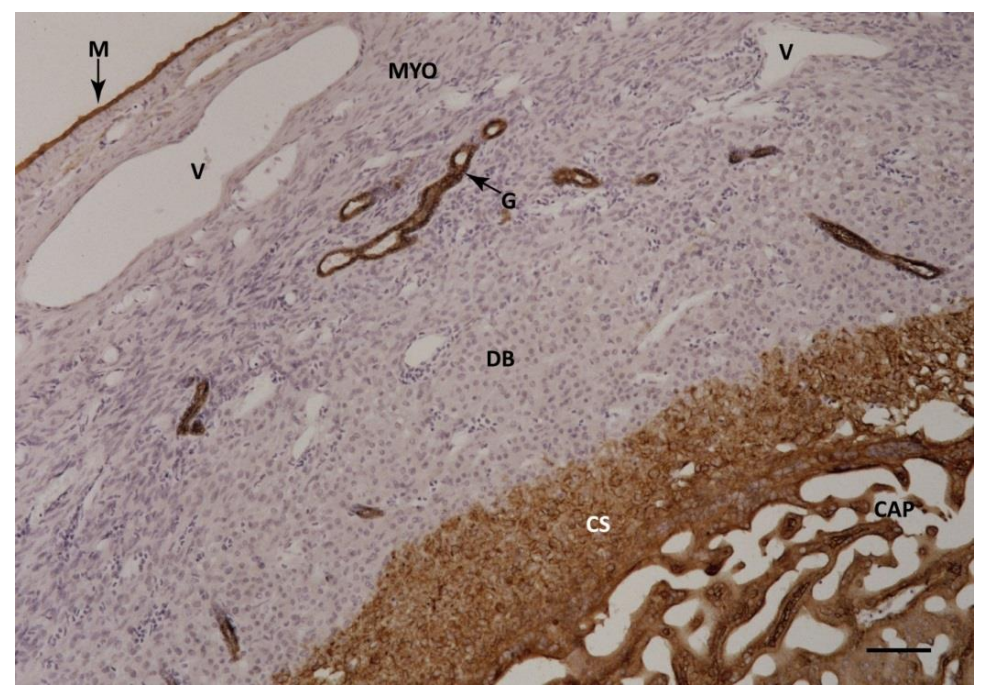

Figure 9. Histological section of uterus obtained from a captive-bred Carollia on day 50 p.c. This animal carried a normal (non-delayed) limb plate stage embryo. This tract was perfusion-fixed, sectioned and then stained immunocytochemically with a monoclonal antibody cocktail (AE1/AE3) directed against a variety of cytokeratins. The only reactive elements in this field are trophoblast within the chorioallantoic placenta (CAP), the cytotrophoblastic shell (CS) on the placenta's periphery, the endometrial glands $(G)$ and the mesothelium (M). DB = decidua basalis. $\mathrm{MYO}=$ myometrium. $\mathrm{V}=$ uterine veins. $B a r=100 \mu \mathrm{m}$. 


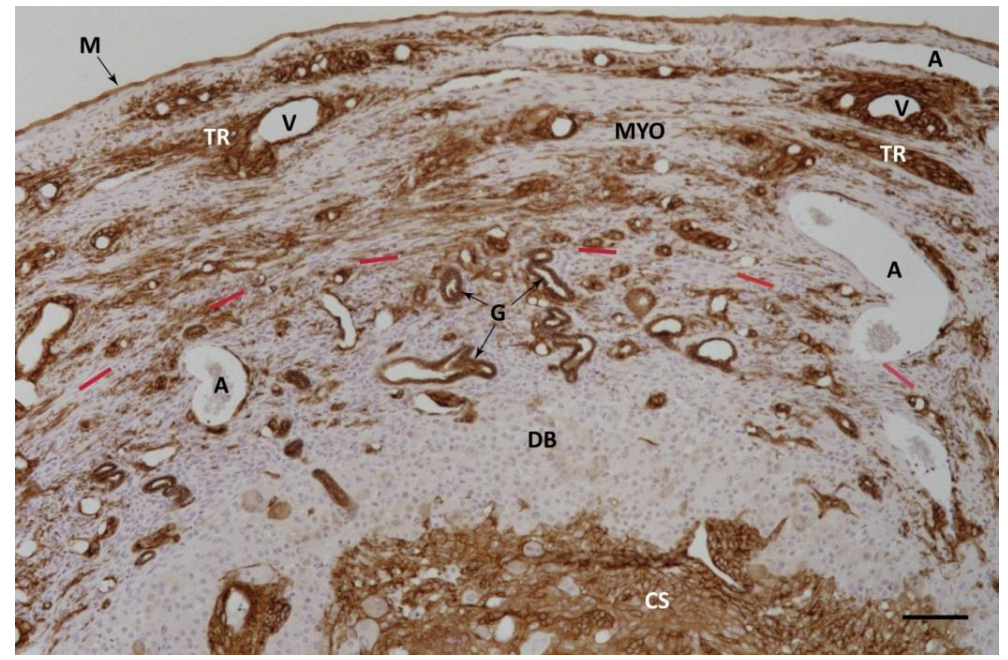

Figure 10. Section of uterus obtained from a captive-bred Carollia on day 60 p.c. This animal carried a primitive streak stage embryo in diapause. This uterus was perfusion-fixed, sectioned and stained immunocytochemically with a monoclonal antibody cocktail (AE1/AE3) directed against a variety of cytokeratins and lightly counterstained with hematoxylin. An abundance of highly invasive trophoblast (TR), that was strongly reactive with the antibody mix, surrounds the maternal capillaries and veins (V) in the myometrium (MYO). The diffuse staining around these vessels was attributable to an abundance of dendritic processes projecting out from the trophoblast.There was only a patchy association of trophoblast with the maternal arteries. The only other reactive elements in this field are trophoblast of the placenta's cytotrophoblastic shell (CS). No endometrial glands (G) were present on the myometrial side of the red bars. $\mathrm{DB}=$ decidua basalis. Modified and reproduced with permission [62]. Bar $=100 \mu \mathrm{m}$.

In placentae serving pregnancies that had progressed beyond the primitive streak stage (i.e., beyond any embryonic diapause), more of the cytotrophoblast had become organized into monolayer sheets of more differentiated cells (Fig. 12). These now lie immediately adjacent to the syncytiotrophoblast lining maternal vascular spaces. These cytotrophoblast cells exhibited apicalbasal polarity, better-developed junctional complexes, and a basal lamina (although the latter not initially). In both normal and post-diapause pregnancies, the invasion of any portion of the developing placenta by vascularized allantoic mesenchyme was always associated with such transformation of the cytotrophoblast. This suggests that the cytotrophoblast may be providing a scaffold for ingrowth of the allantoic mesenchyme, as well as locally stimulating proliferation of its mesenchymal and endothelial cells. It seems relevant to note that endothelial cells in the maternal vessels serving the definitive, chorioallantoic placenta of another bat, $M$. rufus, are the most proliferative ever observed in mammals [68]. This proliferation might well be stimulated in a paracrine fashion by abundant cytotrophoblast in both the developing hemochorial placenta and uteroplacental vessel walls.

These observations indicate that in addition to its widely-recognized proliferative function, placental cytotrophoblast in Carollia may perform important morphogenic roles [25]. Conversely, it would seem that delayed differentiation of the cytotrophoblast might be one way of inhibiting vascularization of the embryonic side of the chorioallantoic placenta and thereby further embryonic development during diapause. 


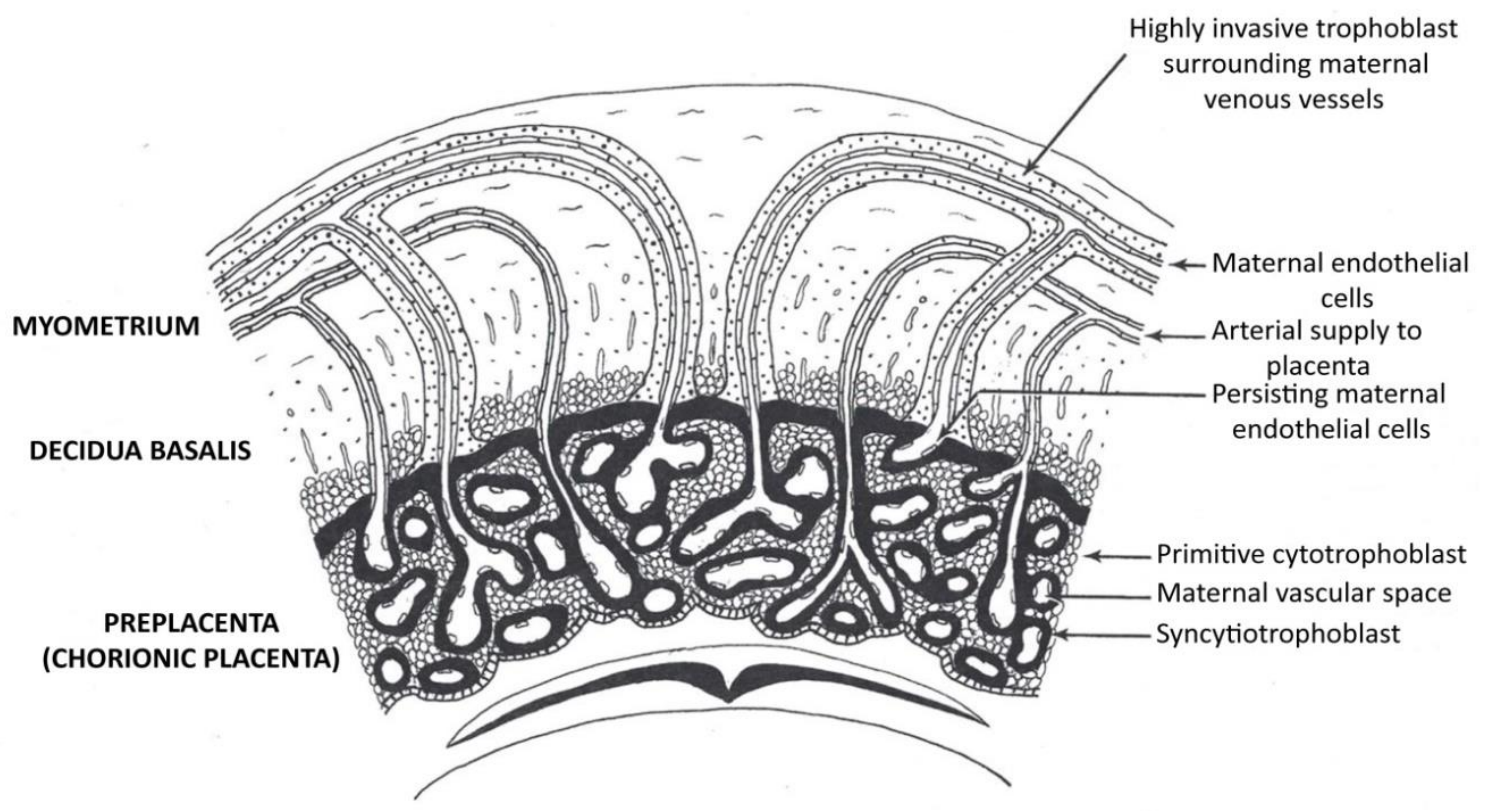

Figure 11. Diagram depicting the organization of the preplacenta serving an embryo in diapause at the primitive streak stage. Modified and reproduced with permission [62].

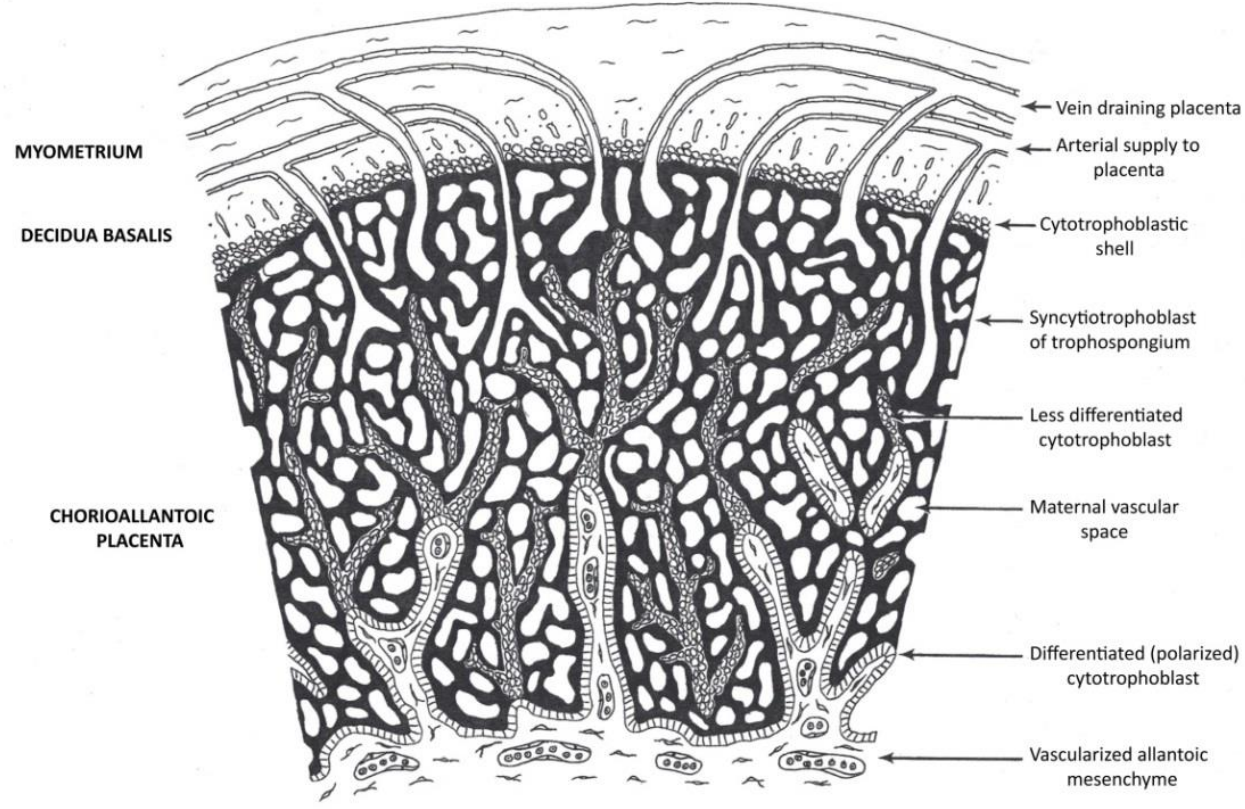

Figure 12. Diagram depicting the organization of an early chorioallantoic placenta serving a limb bud stage embryo that had not spent a period in diapause. Modified and reproduced with permission [62].

Several lines of evidence would seem to support this suggested morphogenetic function of cytotrophoblast: 1) It is well-established that basal laminae serve as substrata for the migration of mesoderm away from the primitive streak and for the movement of mesenchymal cells along epithelia in a variety of other developmental situations [69-71]. 2) When pregnancies are delayed 
in Carollia, both normal cytotrophoblastic differentiation and invasion of the preplacenta by vascularized mesoderm are retarded. 3) During development of the chorioallantoic placenta in Carollia sizeable areas of trophospongium form (Fig. 12). These are then thoroughly invaded by cytotrophoblast and vascularized, allantoic mesenchyme (Fig 13). Since cytotrophoblast had already proliferated to produce the trophospongium, this secondary invasion by cytotrophoblast and its persistence as a continuous layer everywhere in the interhemal barrier until parturition must have other purposes. One of these may be to control and stimulate invasion of those regions by vascularized allantoic mesenchyme [25]. 4) Human cytotrophoblast cells have been found to secrete a variety of angiogenic and vasculogenic factors, e.g. VEGF family members, PIGF and Ang2 [72-74].

Cytotrophoblast in primate placentae may well have the same morphogenetic function as suggested for Carollia [25, 75]. There is, however, no model of human or nonhuman primate placentation in which invasion of the chorioallantoic placenta by allantoic mesenchyme fails to proceed, apparently because of a delay in cytotrophoblastic differentiation. This is an excellent example of how the study of embryonic diapause can provide new insights into the control of a crucial developmental process in our own species. There are, however, important human obstetrical conditions (e.g., preeclampsia and fetal growth retardation) in which some trophoblastic differentiation is abnormal $[74,76]$.

\section{Areas for future investigation}

The nature of the environmental cue(s) regulating seasonal breeding and the timing of the embryonic diapause in Carollia is unknown. Populations living only slightly north of the Equator, such as those in French Guiana (latitude $5^{\circ} \mathrm{N}$ ) and Trinidad (latitude $10-11^{\circ} \mathrm{N}$ ) are exposed to annual changes in daylength [9], but these are small and of unknown physiological significance to this species. There are also seasonal changes in rainfall; however, the onset and termination of rainy seasons are subject to considerable variation and therefore would seem to be unreliable cues for optimally timing annual reproductive cycles in species with long gestation periods. Finally, seasonal food availability and nutritional content are undoubtedly important to these bats, but we lack a good picture of when these may become physiologically limiting. Finally, the neuroendocrine pathways whereby stress and some seasonal factor trigger diapause in Carollia are presumably somewhat different and remain to be elucidated.

In recent years, increasing efforts have been made to define the endocrine and molecular controls of diapause. In the case of bats which exhibit the postimplantational version, this has generally been attempted in the absence of any detailed studies of the placenta mediating exchange between mother and the embryo. This void needs to be filled, because placental components may be critical targets and effectors of the signals controlling diapause. For example, in Carollia there are major morphological and developmental differences between the placentae of normal versus delayed pregnancies. Some of these (e.g., retarded trophoblastic differentiation and vascularization of the embryonic side of the placenta) may play important roles in causing or maintaining the diapause. There may be others that await identification, e.g., possibly a reduced placental transfer of nutrients or production of growth factors, as well as altered or missing embryo signaling of the placenta.

Although a postimplantational diapause has now been found in a few species belonging to at least five different chiropteran families [4, 5], the reproductive biology of this large mammalian order has not been adequately studied. Diapause probably exists in more species, and the physiological mechanisms involved may or may not be similar in relatively unrelated families (e.g., the Phyllostomidae and Pteropodidae). As some members of these two families can be readily maintained in captivity, they may provide useful models for studying placental development and 
function. We presently do not have models, other than Carollia, in which this can be done in normal versus delayed (non-pathological) pregnancies including a diapause. The value of such a comparative approach has been demonstrated by suggestive evidence for new functions of cytotrophoblast found in Carollia.

Finally, the cell biology of the extraordinarily invasive trophoblast discovered in Carollia and the closely-related $D$. youngi warrants further investigation. This is non-neoplastic tissue that is highly invasive, genetically distinct from those of the mother, and develops from precursor trophoblast that undergoes a dramatic epithelio-mesenchymal transformation in response to unidentified signals. In Carollia this has only been observed in pregnancies that are in, or came out of, a diapause and is of unknown functional significance. In contrast, in D. youngi this develops in apparently normal pregnancies, extensively invades arterial vessels supplying the uterus via interstitial migration (i.e., through their perivascular connective tissue), and is intimately associated with their dilatation. Based upon similarities to humans, the latter is presumably essential for healthy progression of the pregnancy. This seems to be caused in large part by a remarkable dedifferentiation of their vascular smooth muscle cells, possibly in response to signalling by the trophoblast, which is followed by their redifferentiation after parturition [61; Badwaik, Rasweiler \& Muradali, unpub. observ.]. In humans, deficient trophoblastic invasion and remodeling of the endometrial spiral arteries play important roles in the pathogenesis of preeclampsia and fetal growth retardation [76, 77].

Animals All animal work discussed in this review, whether conducted in New York or on Trinidad, was approved by the Animal Care and Use Committee of the Weill Medical College of Cornell University. All wild specimens were collected and exported with the permission of the Wildlife Section, Forestry Division of the Ministry of Agriculture, Land and Marine Resources of the Republic of Trinidad and Tobago. Bats maintained at Cornell were housed in centralized animal facilities in accordance with the "Principles of Laboratory Animal Care" (NIH Publication No. 8623). 


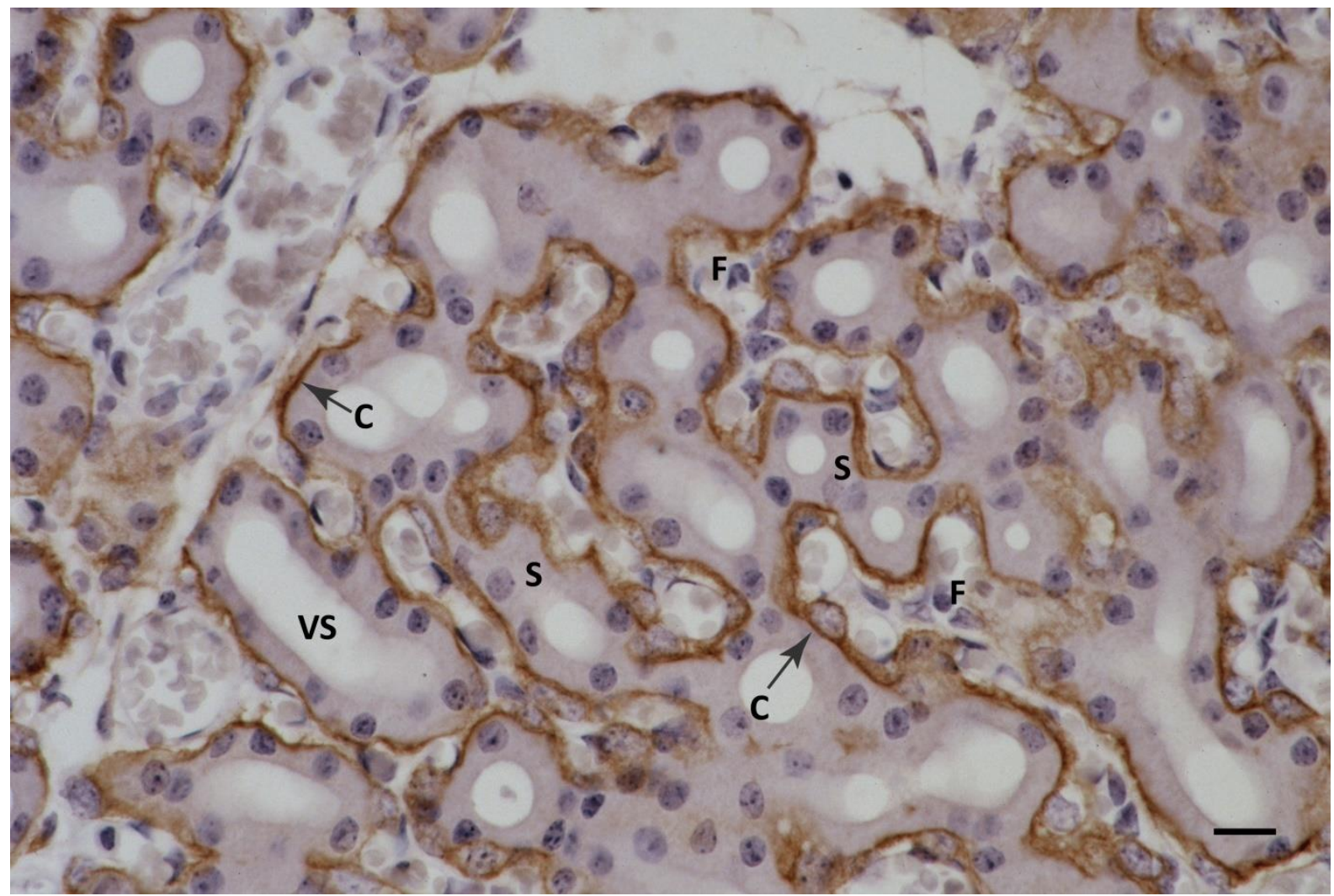

Figure 13. Section of perfusion-fixed, chorioallantoic placenta serving a normal, $18 \mathrm{~mm}$ crown-rump length fetus. This was obtained from a captive-bred Carollia on day 80 p.c. The section was stained immunocytochemically with antibody mix AE1/AE3 to demonstrate the presence of cytokeratins and counterstained with hematoxylin. All of the smallest maternal vascular spaces (VS) were lined by a placental barrier that was hemodichorial. This included a continuous layer of syncytiotrophoblast (S) and an underlying layer of cytotrophoblast (C) lying immediately adjacent to the fetal connective tissue (F). In most of the placenta late in pregnancy, as evident here, cytokeratin expression by the cytotrophoblast was generally strong, while that by the syncytiotrophoblast was very weak. This image demonstrates how cytotrophoblast secondarily invades all of the trophospongium (cf., Figure 12) to establish a hemodichorial barrier everywhere in the definitive placenta. Bar $=25 \mu \mathrm{m}$.

\section{Declaration of interest}

The authors declare that there is no conflict of interest that could be perceived as prejudicing the impartiality of this study.

\section{Funding}

This research was supported by the Department of Obstetrics and Gynecology, Weill Medical College of Cornell University and National Institutes of Health Grant HD-28592. Nilima K. Badwaik was also provided with support and assistance by the Council for International Exchange of Scholars as a Visiting Scholar under the Fulbright Scholar Program.

\section{Acknowledgements}

Thanks go to the staff of the Department of Life Sciences, University of the West Indies, St. Augustine, Trinidad for their generous assistance and the use of departmental facilities during the field work required for these studies. This review is dedicated to the memory of two pioneers of 
comparative reproductive biology and our mentors, Professors A. Gopalakrishna and William A. Wimsatt.

\section{References}

[1] Fenelon JC, Banerjee A \& Murphy BD. 2014 Embryonic diapause: development on hold. International Journal of Developmental Biology 58 163-174

[2] Renfree MB \& Fenelon JC. 2017 The enigma of embryonic diapause. Development 144 3199-3210

[3] Fenelon JC \& Renfree MB. 2018 The history of the discovery of embryonic diapause in mammals. Biology of Reproduction 99 242-251

[4] Rasweiler JJ, IV \& Badwaik NK. 1997 Delayed development in the short-tailed fruit bat, Carollia perspicillata. Journal of Reproduction and Fertility 109 7-20.

[5] Racey PA \& Entwistle C. 2000 Life-history and reproductive strategies of bats. In Reproductive Biology of Bats. Pp 363-414. Eds EG Crichton \& PH Krutzsch. (Academic Press: London)

[6] Meenakumari KJ \& Krishna A. 2005 Cynopterus sphinx. Zoology 108 131-140.

[7] Bates PJJ \& Harrison DL. 1997 Bats of the Indian Subcontinent. (Harrison Zoological Museum: Sevenoaks, Kent, United Kingdom)

[8] Gardner AL. 2007 Mammals of South America. Volume 1. Marsupials, Xenarthrans, Shrews, and Bats. (The University of Chicago Press: Chicago)

[9] https://aa.usno.navy.mil/data/docs/Dur OneYear.php

[10] Rasweiler JJ, IV, Cretekos CJ \& Behringer RR. 2009 The short-tailed fruit bat Carollia perspicillata. A model for studies in reproduction and development. In Emerging Model Organisms. A Laboratory Manual. Volume 1. Pp 519-555. (Cold Spring Harbor Laboratory Press: Cold Spring Harbor, New York)

[11] Cosson JF \& Pascal M. 1994 Stratégie de reproduction de Carollia perspicillata (L., 1758) (Chiroptera, Phyllostomidae) en Guyane Française. Revue d'écologie (la Terre et la Vie) 49 117-137

[12] Badwaik NK, Rasweiler JJ, IV \& Oliveira SF. 1997 Formation of reticulated endoderm, Reichert's membrane, and amniogenesis in blastocysts of captive-bred, short-tailed fruit bats, Carollia perspicillata. The Anatomical Record 247 85-101

[13] Rasweiler JJ, IV, Oliveira SF \& Badwaik NK. 2002 An ultrastructural study of interstitial implantation in captive-bred, short-tailed fruit bats, Carollia perspicillata: trophoblastic adhesion and penetration of the uterine epithelium. Anatomy and Embryology 205 371-391

[14] Kleiman DG \& Davis TM. 1979 Ontogeny and maternal care. In Biology of Bats of the New World Family Phyllostomatidae, Part III. Pp 387-402. Eds RJ Baker, JK Jones, Jr \& DC Carter. (Texas Tech Press: Lubbock, Texas)

[15] Laska M. 1990 Gestation period and between-birth intervals in Carollia perspicillata (Phyllostomatidae, Chiroptera). Journal of Zoology, London 222 697-702

[16] Bonilla H de \& Rasweiler JJ, IV. 1974 Breeding activity, preimplantation development, and oviduct histology of the short-tailed fruit bat, Carollia, in captivity. The Anatomical Record 179 385-404

[17] Rasweiler JJ, IV \& Bonilla H de. 1991 Menstruation in short-tailed fruit bats (Carollia spp.) Journal of Reproduction and Fertility 95 231-248

[18] Rasweiler JJ, IV, Badwaik NK \& Mechineni KV. 2010 Selectivity in the transport of spermatozoa to oviductal reservoirs in the menstruating fruit bat, Carollia perspicillata. Reproduction 140 743-757

[19] Rasweiler JJ, IV, Badwaik NK \& Mechineni KV. 2011 Ovulation, fertilization, and early embryonic development in the menstruating fruit bat, Carollia perspicillata. The Anatomical Record 294 506-519

[20] Napier JE, Caron S, Reavill DR, Murphy H \& Garner MM. 2009 Proliferative endometrial lesions in a group of Seba's short-tailed bats (Carollia perspicillata). Journal of Zoo and Wildlife Medicine 40 437-444

[21] Burns JM, Baker RJ \& Bleier WJ. 1972 Hormonal control of 'delayed development' in Macrotus waterhousii. I. Changes in plasma thyroxine during pregnancy and lactation. General and Comparative Endocrinology 18 54-58 
[22] Burns JM. 1981 Aspects of endocrine control of delay phenomena in bats with special emphasis on delayed development. Journal of Reproduction and Fertility Supplement 29 61-66

[23] Rasweiler JJ, IV. 1973 Care and management of the long-tongued bat, Glossophaga soricina (Chiroptera: Phyllostomatidae), in the laboratory, with observations on estivation induced by food deprivation. Journal of Mammalogy 54 391-404

[24] Rasweiler JJ, IV \& Badwaik NK. 2011 Additional comments on the nutrition and feeding of some phyllostomid bats in the laboratory. In Bats in Captivity. Volume 3: Diet and Feeding - Environment and Housing. Pp 111-117. Ed SM Barnard. (Logos Press: Washington, D.C.)

[25] Badwaik NK \& Rasweiler JJ, IV. 2001 Altered trophoblastic differentiation and increased trophoblastic invasiveness during delayed development in the short-tailed fruit bat, Carollia perspicillata. Placenta 22 124-144

[26] Cretekos CJ, Weatherbee SD, Chen C-H, Badwaik NK, Niswander L, Behringer RR \& Rasweiler JJ, IV. 2005 Embryonic staging system for the short-tailed fruit bat, Carollia perspicillata, a model organism for the mammalian order Chiroptera, based upon timed pregnancies in captive-bred animals. Developmental Dynamics 233 721-738

[27] Rasweiler JJ, IV \& Badwaik NK. 1996 Improved procedures for maintaining and breeding the shorttailed fruit bat (Carollia perspicillata) in a laboratory setting. Laboratory Animals 30 171-181

[28] ffrench R. 1991 A Guide to the Birds of Trinidad \& Tobago, Second Edition. (Comstock Publishing Associates: Ithaca, New York)

[29] Krishna A \& Bhatnagar KP. 2011 Hormones and reproductive cycles in bats. In Hormones and Reproduction in Vertebrates. Pp 241- 289. Eds DO Norris \& HK Lopez. (Elsevier: San Diego)

[30] Rasweiler JJ, IV. 1977 The care and management of bats as laboratory animals. In Biology of Bats, Volume III. Pp 519-617. Ed WA Wimsatt. (Academic Press, New York)

[31] Fleming TH. 1988 The Short-tailed Fruit Bat. (The University of Chicago Press: Chicago)

[32] York HA \& Billings SA. 2009 Stable-isotope analysis of diets of short-tailed fruit bats (Chiroptera: Phyllostomidae: Carollia). Journal of Mammalogy 90 1469-1477

[33] Bohlender EE, Perez-Torres J, Borray-Escalante NA \& Stevens RD. 2018 Dietary variation during reproduction in Seba's short-tailed fruit bat. Journal of Mammalogy 99 440-449

[34] Rasweiler JJ, IV \& Badwaik NK. 1999 Relationships between orientation of the blastocyst during implantation, position of the chorioallantoic placenta, and vascularization of the uterus in the noctilionoid bats Carollia perspicillata and Noctilio albiventris. Placenta 20 241-255

[35] Badwaik NK \& Rasweiler JJ, IV. 2000 Pregnancy. In Reproductive Biology of Bats. Pp 221-293. Eds EG Crichton \& PH Krutzsch. (Academic Press: London)

[36] Oliveira SF, Rasweiler JJ, IV \& Badwaik NK. 2000 Advanced oviductal development, transport to the preferred implantation site, and attachment of the blastocyst in captive-bred, short-tailed fruit bats, Carollia perspicillata. Anatomy and Embryology 201 357-381

[37] Rasweiler JJ, IV. 1993 Pregnancy in Chiroptera. The Journal of Experimental Zoology 266 495-513

[38] Rasweiler JJ, IV. 1979 Differential transport of embryos and degenerating ova by the oviducts of the long-tongued bat, Glossophaga soricina. Journal of Reproduction and Fertility 55 329-334

[39] Rasweiler JJ, IV. 1974 Reproduction in the long-tongued bat, Glossophaga soricina. II. Implantation and early embryonic development. American Journal of Anatomy 139 1-36

[40] Yoshinaga K. 2013 A sequence of events in the uterus prior to implantation in the mouse. Journal of Assisted Reproduction and Genetics 30 1017-1022

[41] Mossman HW. 1987 Vertebrate Fetal Membranes. (Rutgers University Press: New Brunswick, New Jersey)

[42] Enders AC, Liu IKM, Mead RA \& Welsh AO. 1995 Active and passive morphological interactions of trophoblast and endometrium during early implantation. In Molecular and Cellular Aspects of Periimplantation Processes. Pp 168-182. Ed SK Dey. (Springer: Berlin)

[43] Mossman HW. 1937 Comparative morphogenesis of the fetal membranes and accessory uterine 
structures. Contributions to Embryology, The Carnegie Institution of Washington 26 129-246

[44] Marshall AJ. 1949 Pre-gestational changes in the giant fruit bat (Pteropus giganteus), with special reference to an asymmetric endometrial reaction. Proceedings of the Linnean Society of London 161 26-36

[45] Marshall AJ. 1953 The unilateral endometrial reaction in the giant fruit-bat (Pteropus giganteus Brünnich). Journal of Endocrinology 9 42-44

[46] Rasweiler JJ, IV. 1978 Unilateral oviductal and uterine reactions in the little bulldog bat, Noctilio albiventris. Biology of Reproduction 19 467-492

[47] Rasweiler JJ, IV \& Badwaik NK. 2000 The female reproductive tract. In Reproductive Biology of Bats. Pp 157-219. Eds EG Crichton \& PH Krutzsch. (Academic Press: London)

[48] Pow CST \& Martin L. 1994 The ovarian-uterine vasculature in relation to unilateral endometrial growth in flying foxes (genus Pteropus, suborder Megachiroptera, order Chiroptera). Journal of Reproduction and Fertility 101 247-255

[49] Pow CST \& Martin L. 1995 Ovarian and uterine lymphatic drainage in Australian flying-foxes (genus Pteropus, suborder Megachiroptera). Cell and Tissue Research 280 371-381

[50] Gopalakrishna A \& Karim KB. 1971 Localized progestational endometrial reaction in the uterus of the Indian fruit-bat, Rousettus leschenaulti (Desmaret). Current Science 40 490-491

[51] Bernard RTF. 1988 An ovarian rete arteriosum may facilitate reproductive asymmetry in the Egyptian fruit bat (Rousettus aegyptiacus). South African Journal of Science 84 856-857

[52] Heideman PD. 1989 Delayed development in Fischer's pygmy fruit bat, Haplonycteris fischeri, in the Philippines. Journal of Reproduction and Fertility 85 363-382

[53] Heideman PD, Cummings JA \& Heaney LR. 1993 Reproductive timing and early embryonic development in an Old World fruit bat, Otopteropus cartilagonodus (Megachiroptera). Journal of Mammalogy 74 621-630

[54] Heideman PD \& Powell KS. 1998 Age-specific reproductive strategies and delayed embryonic development in an Old World fruit bat, Ptenochirus jagori. Journal of Mammalogy 79 295-311

[55] Rasweiler JJ, IV. 1982 The contribution of observations on early pregnancy in the little sac-winged bat, Pteropteryx kappleri, to an understanding of the evolution of reproductive mechanisms in monovular bats. Biology of Reproduction $27681-702$

[56] Mutere FA. 1967 The breeding biology of equatorial vertebrates: reproduction in the fruit bat, Eidolon helvum, at latitude 0²0' N. Journal of Zoology, London 153 153-161

[57] Rasweiler JJ, IV. 1977 Preimplantation development, fate of the zona pellucida and observations on the glycogen-rich oviduct of the little bulldog bat, Noctilio albiventris. The American Journal of Anatomy 150 269-300

[58] Rasweiler JJ, IV. 1990 Implantation, development of the fetal membranes and placentation in the captive black mastiff bat, Molossus ater. The American Journal of Anatomy 187 109-136

[59] Rasweiler JJ, IV. 1991 Development of the discoidal hemochorial placenta in the black mastiff bat, Molossus ater: evidence for a role of maternal endothelial cells in the control of trophoblastic growth. The American Journal of Anatomy 191 185-207

[60] Kalanithi LEG, Illuzzi JL, Nossov VB, Frisbæk L, Abdel-Razeq, Dopel JA \& Norwitz ER. 2007 Intrauterine growth restriction and placental location. Journal of Ultrasound Medicine 26 1481-1489

[61] Badwaik NK, Rasweiler JJ, IV \& Muradali F. 1998 Co-expression of cytokeratins and vimentin by highly invasive trophoblast in the white-winged vampire bat, Diaemus youngi and the black mastiff bat, Molossus ater, with observations on intermediate filament proteins in the decidua and intraplacental trophoblast. Journal of Reproduction and Fertility 114 307-325

[62] Rasweiler JJ, IV, Badwaik NK \& Sugarbaker TA. 2000 Unusual patterns of intermediate filament protein expression by the trophoblast and decidual cells of the short-tailed fruit bat, Carollia perspicillata. Placenta 21 853-869

[63] Hay ED. 1995 An overview of epithelio-mesenchymal transformation. Acta Anatomica 154 8-20

[64] Greenberg G \& Hay ED. 1982 Epithelia suspended in collagen gels can lose polarity and express 
characteristics of migrating mesenchymal cells. Journal of Cell Biology 95 333-339

[65] Greenberg G \& Hay ED. 1986 Cytodifferentiation and tissue phenotype change during transformation of thyroid epithelium to mesenchyme-like cells. Developmental Biology 115 363-379

[66] Greenberg G \& Hay ED. 1988 Cytokeratin and thyroglobulin expression change during transformation of thyroid epithelium to mesenchyme-like cells. Development 102 605-622

[67] Badwaik NK \& Rasweiler JJ, IV. 1998 The interhemal barrier in the chorioallantoic placenta of the greater mustache bat, Pteronotus parnellii, with observations on amplification of its intrasyncytial lamina. Placenta 19 391-401

[68] Rasweiler JJ, IV, Badwaik NK, Salame G \& Abulafia O. 2011 Endothelial hyperproliferation and stratification in uteroplacental blood vessels of the black mastiff bat, Molossus rufus. Placenta 32 633-644

[69] Hay ED. 1978 Role of basement membranes in development and differentiation. In Biology and Chemistry of Basement Membranes. Pp 119-136. Ed N Kefalides. Academic Press: New York

[70] Hay ED. 1985 Interaction of migrating embryonic cells with extracellular matrix. Experimental Biology and Medicine 10 174-193

[71] Sanders EJ. 1986 Mesoderm migration in the early chick embryo. In Developmental Biology. A Comprehensive Synthesis. Volume 2. Pp 449-480. Ed L Browder. Plenum Publishing: New York

[72] Ghosh D, Sharkey AM, Charnock-Jones DS, Dhawan L, Dhara S, Smith SK \& Sengupta J. 2000 Expression of vascular endothelial growth factor (VEGF) and placental growth factor (PIGF) in conceptus and endometrium during implantation in the rhesus monkey. Molecular Human Reproduction 6 935-941

[73] Zhou Y, Bellingard V, Feng K-T, McMaster M \& Fisher SJ. 2003 Human cytotrophoblasts promote endothelial survival and vascular remodeling through secretion of Ang2, PIGF, and VEGF-C. Developmental Biology 263 114-125

[74] Fisher SJ. 2004 The placenta problem: linking abnormal cytotrophoblastic differentiation to the maternal symptoms of preeclampsia. Reproductive Biology and Endocrinology 253

[75] Mori M, Ishikawa, G, Luo S-S, Mishima T, Goto T, Robinson JM, Matsubara S, Takeshita T, Kataoka H \& Takizawa T. 2007 The cytotrophoblast layer of human chorionic villi becomes thinner but maintains its structural integrity during gestation. Biology of Reproduction 76 164-172

[76] Fisher SJ. 2015 Why is placentation abnormal in preeclampsia? American Journal of Obstetrics \& Gynecology 213, Issue 4, Supplement S115-S122

[77] Burton GT. 2018 Pathophysiology of placental-derived fetal growth restriction. American Journal of Obstetrics \& Gynecology 218, Issue 2, Supplement S745-S761

\section{Audience Discussion}

Katarina Jewgenow: Does a stress event cause the delay of pregnancy? Is it just random or do they need specific signals of stress? Do you have any idea?

John Rasweiler: Frankly, I can't make biological sense out of this. I do not think stress plays any role in triggering the seasonal diapause in the wild. The diapause of variable length frequently observed in our captive animals may have been a stress response to experimental conditions. We tried to stimulate a diapause of more consistent length by food depriving captive females once a week during pregnancy, but what we observed looks much like the stress response (i.e., a wide range of gestation lengths). Furthermore, out in the wild at the time of the year when pregnancies go into a diapause, the habitat is lush and green, and there is an abundance of food. As Carollia can shift back and forth between fruit and insects, I do not see food deprivation as being a reliable trigger for the natural, seasonal delay. By taking early pregnancies into a diapause in the wild, this species may actually be anticipating nutritional demands to be faced many months later, when females are in advanced pregnancy, neonatal young are growing rapidly, or adult females are simultaneously lactating and pregnant. Were wild females to respond to some temporary stressor by taking early pregnancies into a diapause, their births might eventually occur at less optimal times. Finally, our observations would seem to have an important lesson for others who work with wild animals in captivity; wild animals may sometimes respond quite differently in experimental situations than domesticated species. 